\title{
High-resolution daily gridded data sets of air temperature and wind speed for Europe
}

\author{
Sven Brinckmann ${ }^{1}$, Stefan Krähenmann ${ }^{2}$, and Peter Bissolli ${ }^{1}$ \\ ${ }^{1}$ Climate Monitoring, Deutscher Wetterdienst, Frankfurter Strasse 135, 63067 Offenbach, Germany \\ ${ }^{2}$ Central Climate Office, Deutscher Wetterdienst, Frankfurter Strasse 135, 63067 Offenbach, Germany \\ Correspondence to: S. Brinckmann (sven.brinckmann@dwd.de)
}

Received: 30 June 2015 - Published in Earth Syst. Sci. Data Discuss.: 12 August 2015

Revised: 22 August 2016 - Accepted: 8 September 2016 - Published: 14 October 2016

\begin{abstract}
New high-resolution data sets for near-surface daily air temperature (minimum, maximum and mean) and daily mean wind speed for Europe (the CORDEX domain) are provided for the period 2001-2010 for the purpose of regional model validation in the framework of DecReg, a sub-project of the German MiKlip project, which aims to develop decadal climate predictions. The main input data sources are SYNOP observations, partly supplemented by station data from the ECA\&D data set (http://www.ecad.eu). These data are quality tested to eliminate erroneous data. By spatial interpolation of these station observations, grid data in a resolution of $0.044^{\circ}(\approx 5 \mathrm{~km})$ on a rotated grid with virtual North Pole at $39.25^{\circ} \mathrm{N}, 162^{\circ} \mathrm{W}$ are derived. For temperature interpolation a modified version of a regression kriging method developed by Krähenmann et al. (2011) is used. At first, predictor fields of altitude, continentality and zonal mean temperature are used for a regression applied to monthly station data. The residuals of the monthly regression and the deviations of the daily data from the monthly averages are interpolated using simple kriging in a second and third step. For wind speed a new method based on the concept used for temperature was developed, involving predictor fields of exposure, roughness length, coastal distance and ERA-Interim reanalysis wind speed at $850 \mathrm{hPa}$. Interpolation uncertainty is estimated by means of the kriging variance and regression uncertainties. Furthermore, to assess the quality of the final daily grid data, cross validation is performed. Variance explained by the regression ranges from 70 to $90 \%$ for monthly temperature and from 50 to $60 \%$ for monthly wind speed. The resulting RMSE for the final daily grid data amounts to $1-2 \mathrm{~K}$ and $1-1.5 \mathrm{~m} \mathrm{~s}^{-1}$ (depending on season and parameter) for daily temperature parameters and daily mean wind speed, respectively. The data sets presented in this article are published at doi:10.5676/DWD_CDC/DECREG0110v2.
\end{abstract}

\section{Introduction}

In climate research, data of meteorological observations are preferably provided in the form of continuous regular grids. In this way the data can be used for regional or global climate monitoring as well as for a comparison with the outputs from numerical weather prediction models and climate models. One of the main and most reliable initial data sources are measurements taken at ground station networks like SYNOP (synoptic observations recommended by the World Meteorological Organization, WMO). Interpolation or averaging procedures are used to transform such point data to values representative for grid cells of regular size and distance.
For the near-surface temperature a variety of gridded observational data is available. One of the most prominent global data sets is the HadCRUT4 data set provided by the UK Met Office Hadley Centre and the Climatic Research Unit (CRU) at the University of East Anglia (Morice et al., 2012), covering monthly values in $5^{\circ}$ spatial resolution since 1850. In recent years several more global temperature data sets have been published (e.g., Smith et al., 2008; Hansen et al., 2010; Rohde et al., 2013). On a regional scale, temperature data sets with higher resolutions in space (e.g., Hogewind and Bissolli, 2011) and time have been produced. For Europe the E-OBS project has set up an archive of grid- 
ded daily data in a horizontal resolution of $0.25^{\circ}$, starting in 1950 (Haylock et al., 2008). Since regional models increasingly realize even higher resolutions, reference data sets with resolutions below $0.25^{\circ}$ are more and more requested. So far, such data are only produced on a national or sub-regional scale.

In addition to the pure observational data sets, so-called reanalysis data represent alternative sources. Measurements from surface stations, radiosondes and satellite data of different meteorological parameters serve as input data for the assimilation scheme of weather prediction models. Threedimensional gridded data for a variety of parameters describing the initial status of the atmosphere are obtained. However, the dependency on model physics makes reanalysis data unsuitable for the evaluation of model forecasts.

Concerning the near-surface wind speed, the availability of gridded observational data is currently very low. On a national level a few efforts have been made to calculate horizontal wind fields based on station reports (e.g., Luo et al., 2008; Gerth and Christoffer, 1994; Walter et al., 2006). For larger regions like Europe no such data fields are available at present.

Within DecReg (decadal regional predictability), a subproject of MiKlip (decadal climate predictions), the predictive skill of regional climate models on a decadal timescale is investigated using hindcast experiments. Independent gridded observational data sets for the European region are used as reference. In order to meet the spatial scale of these models $(\approx 7 \mathrm{~km}$ for the COSMO-CLM model; http://www. clm-community.eu/), new reference data with resolutions below the $25 \mathrm{~km}$ realized by E-OBS are required. This request represents a big challenge because the grid size is limited by the density of station observations. More complex interpolation methods are needed to maintain a certain quality on a relatively fine grid. As contribution to DecReg, the Deutscher Wetterdienst (DWD) aims to provide gridded observational data of daily temperature and wind speed in high resolution for the time period 1961-2010.

A variety of interpolation methods can be used to derive continuous field data based on point measurements (see overview given by, for example, Li and Heap, 2008). In cases of relatively coarse grid sizes (with a high number of samples per grid cell) simple averaging techniques are sufficient. For relatively fine grids the value at a given point has to be estimated on the basis of information of surrounding data points. Deterministic interpolation methods are based on the assumption of a certain function, which describes the spatial changes of the target variable. For example, linear regression on the coordinates $x$ and $y$ using least-squares fitting represents a simple form of a deterministic interpolator. A very prominent deterministic approach is the inverse distance weighting method (IDW), in which the information of nearby stations is used to determine the value at certain target coordinates, expecting a decrease of influence with increasing distance. An interpolation method involving proba- bilistic elements (often called a stochastic or geostatistic approach) is so-called kriging (after Daniel G. Krige, further developed by Matheron, 1963). Compared to IDW, in kriging the geographic distribution of the surrounding data points is considered (solving the cluster problem) and the weights are optimized by considering the spatial correlation observed for the target variable. In contrast to deterministic methods, kriging directly provides uncertainty estimates for each grid point. Many meteorological parameters depend on certain local characteristics (like temperature and pressure on the altitude or wind speed on the surrounding vegetation and topography). Such secondary information can be included in the interpolation procedure by a regression approach.

In this work a combination of regression and kriging is used to compute gridded data in $0.044^{\circ}(\approx 5 \mathrm{~km})$ horizontal resolution of daily mean $10 \mathrm{~m}$ scalar wind speed and daily $2 \mathrm{~m}$ air temperatures (minimum, maximum and mean). Here, the results for the decade 2001-2010 are presented. Earlier decades are planned to be added to this new data record in the future.

\section{Input data}

\subsection{Data sources}

The main input data sources used in this work are SYNOP reports. Daily minimum temperatures $\left(T_{\min }\right.$, observed between 18:00 and 06:00 UTC) and daily maximum temperatures ( $T_{\max }, 06: 00$ to $18: 00$ UTC) are taken from the daily SYNOP reports at 06:00 and 18:00 UTC, respectively. For the calculation of daily mean temperature $\left(T_{\mathrm{avg}}\right)$ and daily mean wind speed $\left(V_{\text {avg }}\right)$, hourly reports (current temperature; 10 min before time of synoptic message; wind speed averaged over last $10 \mathrm{~min}$ before time of message) between 00:00 to 00:00 UTC of the following day are used. The European Climate Assessment \& Dataset project (ECA\&D, Klein Tank et al., 2002) offers additional station measurements for Europe and North Africa (data and metadata available at http://www.ecad.eu). The 62 participating countries have provided quality-checked data records for numerous weather stations. For temperature the ECA\&D record is used to extend the input data in areas with low coverage by SYNOP stations. Due to the issue of partly deviating daily observation periods for the three parameters (a discussion will follow in Sect. 2.3), we apply a selection algorithm for the inclusion of ECA\&D data. In this way we aim to avoid inconsistencies with the input data based on SYNOP. The accuracy of the meteorological data from both archives is limited by the precision of the reports (i.e., number of decimal places), which typically depends on the underlying measurement accuracy. Thus, basic uncertainties of $0.1 \mathrm{~K}$ and approx. $0.5 \mathrm{~m} \mathrm{~s}^{-1}$ (1 knot) can be assumed for the two parameters. 


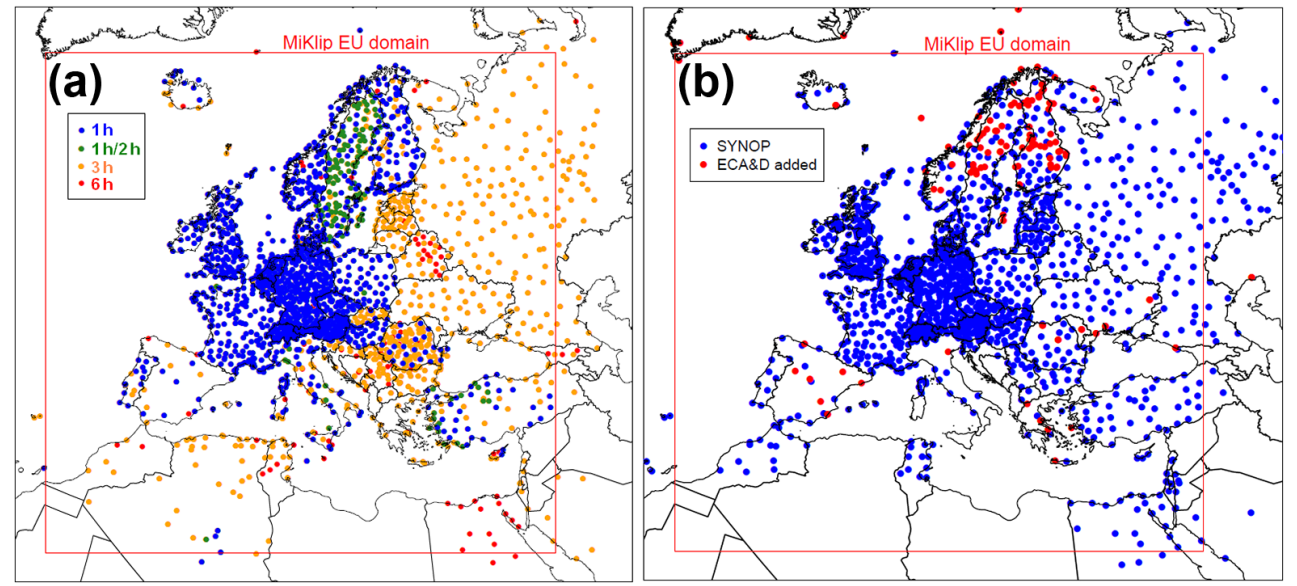

Figure 1. (a) SYNOP stations with hourly data in target DecReg EU domain for January 2010. The color code indicates the frequency of reports (between 1 and $6 \mathrm{~h}$ ). The station records marked green contain hourly data but show gaps for the main dates: 00:00, 03:00, 06:00 UTC, etc. (b) SYNOP data for $T_{\mathrm{avg}}$ (blue) in January 2001 and added ECA\&D data using selection algorithm described in the text.

\subsection{Hourly SYNOP reports}

Figure 1a shows the distribution of SYNOP stations reporting at least every $6 \mathrm{~h}$ (see color code for different frequencies) throughout the target domain for January 2010. In total, 2230 stations are found. Most of the regions in the target domain (indicated by the red frame) are well covered; only for Africa are larger regions without data identified.

Daily $T_{\text {avg }}$ and $V_{\text {avg }}$ are derived by averaging the available hourly data, while assigning only half of the regular weight to the measurements at 00:00 UTC (of current and following day). Depending on the availability of hourly data, daily sample sizes vary between 25 (1-hourly, type 1), 16 (1-/2hourly in Sweden, type 2), 9 (3-hourly, type 3) and 5 (6hourly, type 4). To evaluate the consistency between daily means based on different sample sizes, these values were compared at stations with full daily records of 25 samples. In Fig. 2 daily means of types 3 and 4 in January 2001 are compared with type 1 for temperature (Fig. 2a1, a2) and wind speed (Fig. 2b1, b2) respectively. For temperature we find a high consistency of daily means even for type 4 (Fig. 2a2), with a standard deviation of the error of $0.21 \mathrm{~K}$. For wind speed the precision in the determination of the daily mean decreases considerably with the number of samples per day $\left(\sigma=0.40 \mathrm{~m} \mathrm{~s}^{-1}\right.$ for type 4$)$. However, compared to the relatively high basic uncertainty of $0.5 \mathrm{~m} \mathrm{~s}^{-1}$ for wind speed data and the strong influence of, for example, local roughness conditions on the measurements, these potential discrepancies are found acceptable. For daily means of type 1 up to two missing or non-valid data in a daily record of 25 values were accepted. In the calculation of the daily means these gaps were filled by the mean of the two adjacent dates. Daily records of types $2-4$ were rejected when missing values or inhomogeneities (see Sect. 2.4) occurred.
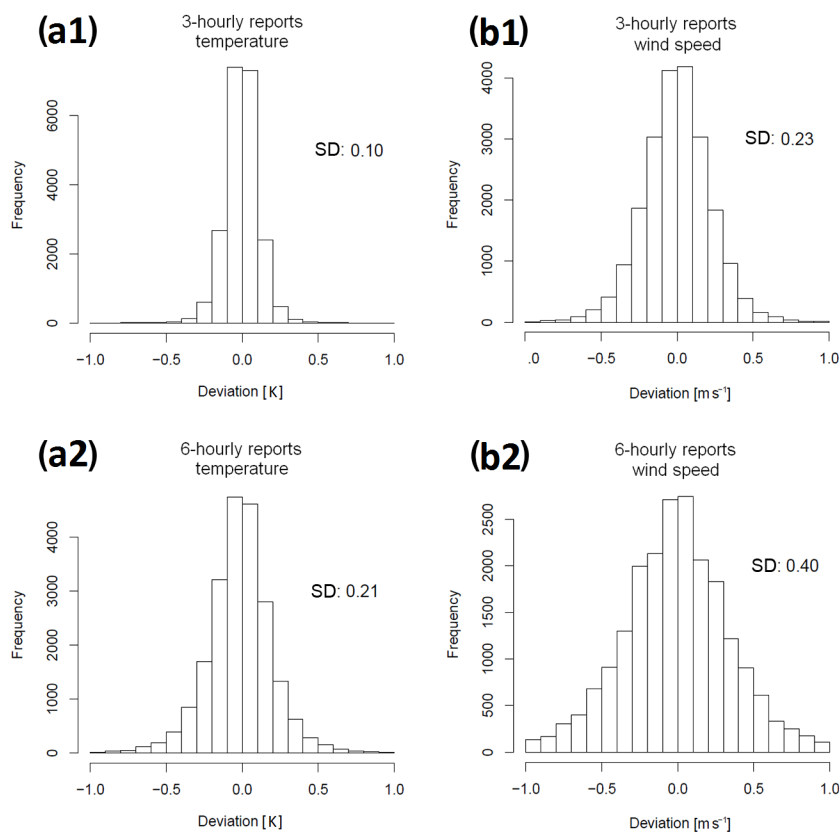

Figure 2. Accuracy of daily mean temperatures (a1, a2) and daily mean wind speeds $(\mathbf{b 1 , ~ b 2 ) ~ i n ~ J a n u a r y ~} 2001$ for different frequencies of observation ( 3 and $6 \mathrm{~h}$ ) using 1-hourly data as reference. The standard deviations, denoted SD, are added to the histograms.

\subsection{Integration of ECA\&D archive for temperature}

The national weather agencies contributing data to ECA\&D determined daily temperature parameters based on partly deviating observation intervals. This leads to potential discrepancies between SYNOP (using consistent intervals; see Sect. 2.1 and 2.2) and ECA\&D, as illustrated in Fig. 3 for January 2010. Daily data from both archives were compared at stations with identical coordinates and altitudes. In 

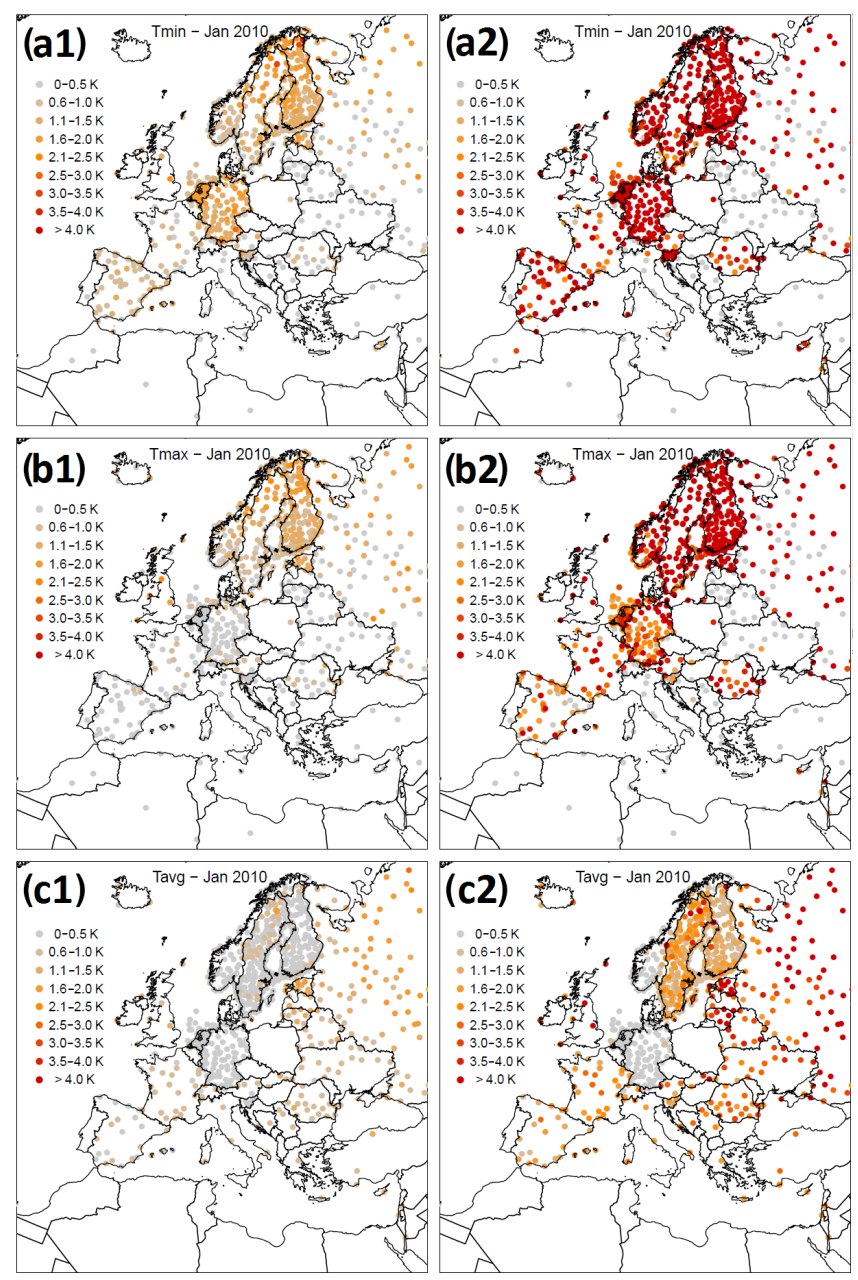

Figure 3. Differences of daily temperature data from SYNOP and ECA\&D for January 2010 at stations with identical coordinates. Mean and maximum absolute daily deviation for minimum temperature (a1, a2), maximum temperature (b1, b2) and mean temperature $(\mathbf{c 1}, \mathbf{c 2})$.

panels a1 and b1 the mean and maximum absolute differences between daily $T_{\min }$ according to ECA\&D and SYNOP are shown. For most of the countries, observation intervals in ECA\&D (in many cases $24 \mathrm{~h}$ intervals, e.g., 00:00 to 00:00 UTC or 06:00 to 06:00 UTC; van den Besselaar et al., 2012) disagree with the $12 \mathrm{~h}$ period in SYNOP, which results in maximum daily deviations of more than $4 \mathrm{~K}$ at most of these stations in this winter month. In contrast, the ECA\&D data of some countries in eastern and southern Europe are indicated to be derived consistently with SYNOP. Qualitatively similar results are found for $T_{\max }$ (Fig. $3 \mathrm{~b} 1$ and b2). For $T_{\text {avg }}$ (Fig. 3c1 and c2) the consistency of both data sources is high in the Netherlands and Germany (00:00 to 00:00 UTC in both archives). In all other countries, deviating intervals and/or calculation methods are apparent for ECA\&D. Overall, root mean square deviations (RMSD) of $1.9 \mathrm{~K}\left(T_{\min }\right)$, $1.7 \mathrm{~K}\left(T_{\max }\right)$ and $0.9 \mathrm{~K}\left(T_{\mathrm{avg}}\right)$ are found in January $2010 . \mathrm{In}$ spring and summer RMSD are usually smaller $(0.8,1.1$ and $0.6 \mathrm{~K}$ for $T_{\min }, T_{\max }$ and $T_{\text {avg }}$, respectively, in July 2010), as a result of increased insolation and more pronounced daily cycles.

To take account of this consistency problem, an algorithm was designed to include suitable data from the ECA\&D archive, considering the density of SYNOP reports in each target area and by data comparison at identical stations (with same coordinates and altitudes). The differences at these stations were used as indicator for the consistency in the target region for each day. Depending on the presence of SYNOP station data in a certain area, different thresholds for considering or rejecting ECA\&D station data were used (e.g., daily deviation required to be smaller than $1 \mathrm{~K}$ if at least one SYNOP station found within radius of $0.75^{\circ}(\approx 80 \mathrm{~km})$ ). In cases where no SYNOP data were available in an area of $\pm 3^{\circ}(\approx 330 \mathrm{~km})$ around a target ECA\&D station and no comparison was possible in a somewhat larger area of $\pm 4.5^{\circ}$ $(\approx 500 \mathrm{~km})$, ECA\&D station data were included without any further testing. In such distances to other data points (about half of the ranges shown in Table 3; compare discussion on variogram ranges in Sect. 3.2), independent station data add essential information to the data field.

In Fig. 1b the station coordinates of a combined data set of SYNOP (blue) and ECA\&D (red) for $T_{\text {avg }}$ in January 2001 are shown. The chosen ECA\&D data add valuable information in Scandinavia, Spain and Greece. The total station number is increased here by about 100 .

The temporal evolution during 2001-2010 of input data for the different parameters is illustrated in Fig. 4. The dotted lines show the fraction of SYNOP reports; the solid lines display the total number of station records used. For wind (grey curve) only SYNOP reports were used, as the number of wind data archived in ECA\&D is currently very low. Towards earlier years the availability of SYNOP reports decreases. This decrease is considerable for the Scandinavian region and for many countries around the Mediterranean Sea (compare Fig. 1a and b). ECA\&D data contribute especially in these early years. The availability of SYNOP data is usually higher for $T_{\min }$ and $T_{\max }$ than for the daily mean values $T_{\text {avg }}$ and $V_{\text {avg }}$.

\subsection{Quality control and assurance}

All input data (the hourly data of each day and the daily data of each month) were quality checked regarding different types of inhomogeneities: (1) outliers, (2) significant shifts in the time series, (3) constant data over longer intervals and (4) exceedance of climatological thresholds. In the following a closer description of the strategies for the example of hourly temperature data is given.

For type 1 the minima and maxima of each daily cycle at a target station are subtracted from the mean of the cycle while omitting the extremes in the averaging. These test values (denoted dtn and dtx in the program) are considered 


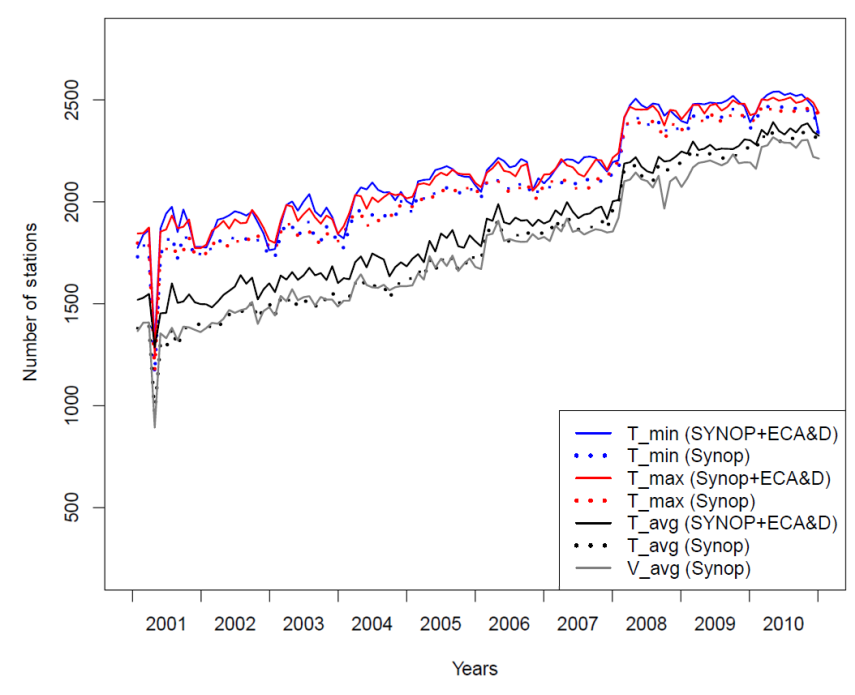

Figure 4. Temporal evolution of total input data used for the interpolations 2001-2010 (see color code). The dotted curves show the basic number made up by SYNOP stations. The differences indicate the increase by the inclusion of ECA\&D data. SYNOP data are used for the interpolations for wind speed only.

absolutely (adding notation 1) and relatively (divided by the standard deviation of the daily values without extremum, notation 2). Based on experiments with data of several example months, empiric thresholds were determined to decide whether a value is considered an outlier or not. Depending on the number of the daily values and the comparison of absolute vs. relative test value these thresholds range between 8 and $14 \mathrm{~K}$ for $\mathrm{d} t n_{1} / \mathrm{d} t x_{1}$ and 4 and 8 for $\mathrm{d} t n_{2} / \mathrm{d} t x_{2}$.

For inconsistencies of types 2 and 3 a running standard deviation $\left(\mathrm{SD}_{\mathrm{r}}\right)$ of five consecutive measurements is calculated. If the minimum of $\mathrm{SD}_{\mathrm{r}}$ (denoted $\mathrm{SD}_{\mathrm{n}}$ in the program) reaches zero, at least five identical values in a row are indicated. For a daily series of in total five values (6-hourly data), such an event is highly unlikely and therefore considered a result of erroneous data. In the case of hourly reports the threshold for a rejection is increased to nine identical values in a row. Our sample data showed that certain conditions in winter allow nearly unchanged temperatures over several hours. Also, the change of $\mathrm{SD}_{\mathrm{r}}$ for each time step is recorded (the maximum difference is denoted $\mathrm{dSD}_{\mathrm{x}}$ ) to identify sudden shifts in the common temperature level. Corresponding tests with data of several example months indicated clear inhomogeneities for dsdx above $7 \mathrm{~K}$. Climatological thresholds were determined month-wise for 19 subregions using the full ECA\&D archive for 1961-2010. The subregions were defined by combining countries of similar climate. For the Scandinavian countries and Russia an additional separation by latitude was applied. If subregions were insufficiently represented by ECA\&D stations, the upper and lower thresholds were slightly increased and decreased, respectively, to achieve realistic temperature limits. Additionally, an adjustment of temperature with al-

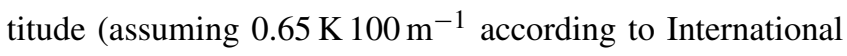
Standard Atmosphere) is carried out to consider stations of high altitude possibly not represented by corresponding data of ECA\&D.

In the case of wind speed, similar techniques, but with adjusted thresholds, were developed to consider erroneous data. Concerning climatological thresholds, a global upper value of $65 \mathrm{~m} \mathrm{~s}^{-1}$ for hourly data was defined. Here, an approach considering season and region is not helpful, as strong wind events can occur in all regions and throughout the year.

For the time series of daily values similar tests following the strategies for the hourly data are applied. This quality check is particularly important for the SYNOP extreme values and for all ECA\&D data because related hourly data are often not available. For stations where both extreme values and hourly raw data are available, a consistency check between extremes based on these hourly data and the aggregated extremes (denoted "hrl" and "agg" in the following) is made (e.g., minimum $T_{\text {min }}^{\text {agg }}$ is not expected to be above minimum of corresponding hourly data $T_{\mathrm{min}}^{\mathrm{hrl}}$ ). For temperature the consistency between the three parameters $T_{\min }, T_{\max }$ and $T_{\text {avg }}$ (e.g., $T_{\min }$ expected to be smaller than $T_{\text {avg }}$ ) is checked for each day, if available.

The hourly data are also used to fill gaps in monthly time series of the extremes, if the overlap between, for example, $T_{\min }^{\mathrm{agg}}$ and $T_{\min }^{\mathrm{hrl}}$ is sufficiently long (more than 10 data points in a month) and the maximum discrepancy between these series is below $2 \mathrm{~K}$. The estimates from the hourly raw data (e.g., $\left.T_{\mathrm{min}}^{\mathrm{hrl}}\right)$ are corrected by the mean deviation between both monthly time series to replace missing data of, for example, $T_{\min }^{\text {agg }}$. Similarly, ECA\&D data are used for filling up incomplete monthly SYNOP series if the same coordinates and a high consistency (maximum deviation below $1 \mathrm{~K}$ ) are found.

During the interpolation process a monthly background field for each parameter is first created. Therefore, only time series with no more than six missing values within a considered month are used. To achieve a more precise estimate of the monthly mean, missing values are reconstructed by linear regression with neighboring stations (depending on available stations search radii increased stepwise to a maximum of $\pm 2.5^{\circ}, \approx 280 \mathrm{~km}$ ). If no suitable stations in the neighborhood are detected and the number of missing data is two or smaller, the mean of the values for the two adjacent dates is used to fill a gap. The values reconstructed in this way are only used to determine the monthly means. For the daily interpolation step, missing data are left unchanged because the interpolation scheme is expected to reproduce missing data more accurately than the rough assumptions used here for the calculation of monthly means. 


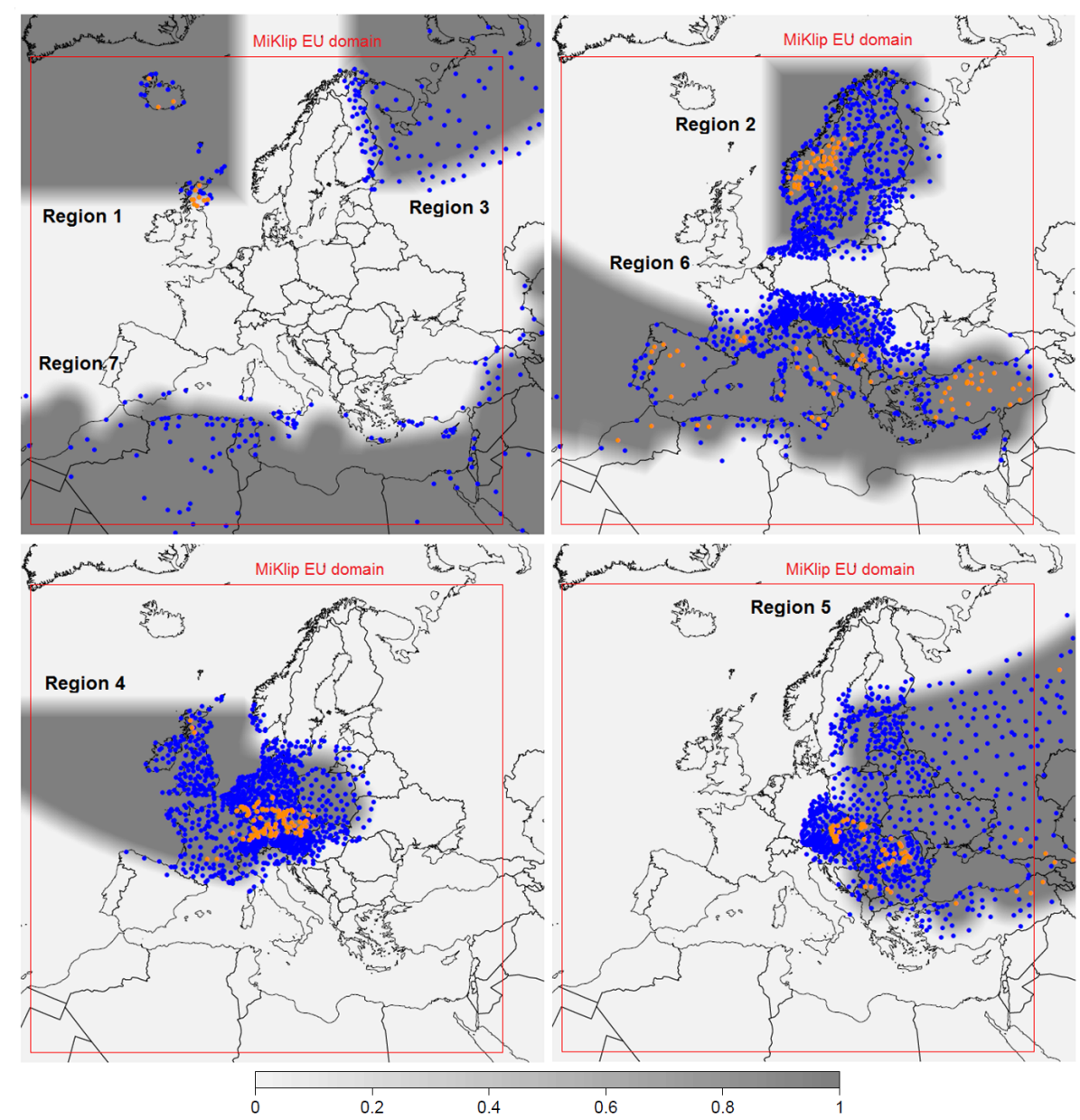

Figure 5. The seven regions used for temperature interpolation. The regional fields are finally merged using the regional weights (grey color scale). Stations used to calculate regional lapse rates are orange.

\section{Interpolation procedure temperature}

For temperature interpolation, a regression kriging approach (strategy proposed by Ahmed and de Marsily, 1987, and Odeh et al., 1995) adapted from Krähenmann et al. (2011) is used. The interpolation is done in four steps: a regression of station monthly means depending on three predictor parameters (altitude, continentality and zonal monthly mean temperatures), followed by interpolation of the regression residuals using kriging to obtain gridded monthly means, a daily adjustment of regression on altitude and, finally, the kriging of daily deviations from station monthly means.

The steps are performed separately in seven overlapping regions (see Fig. $5 ; 2.5^{\circ}, \approx 280 \mathrm{~km}$, overlap). The separation roughly follows the climate classification after Köppen and Geiger (e.g., Sanderson, 1999), and thus relatively homogeneous conditions for temperature are expected within each region. Compared to Krähenmann et al. (2011), slight modifications were made in the partitioning of the regions in order to adapt them to the DecReg domain dealt with in this work.
By considering regions instead of the whole domain, a better adjustment of the regression model and of the kriging parameters (a closer description will follow) during interpolation is achieved. The regional temperature fields of the seven regions are finally merged by linear weighting in the overlap areas (see Fig. 5). This procedure ensures a continuous transition of the data fields between two regions.

\subsection{Regression}

As the first step, a multiple linear regression of the monthly means for each station against data fields of altitude (using elevation data from the shuttle radar topography mission (SRTM; see http://dds.cr.usgs.gov/srtm/version2_1/ SRTM3/) - above $60^{\circ} \mathrm{N}$ complemented by data from the United States Geological Survey (USGS; see https://ta. cr.usgs.gov/GTOPO30) - continentality (after Gorczynski, 1920) and zonal monthly mean temperature (climatology 1961-1990 based on CRU TS 3.00; Mitchell and Jones, 

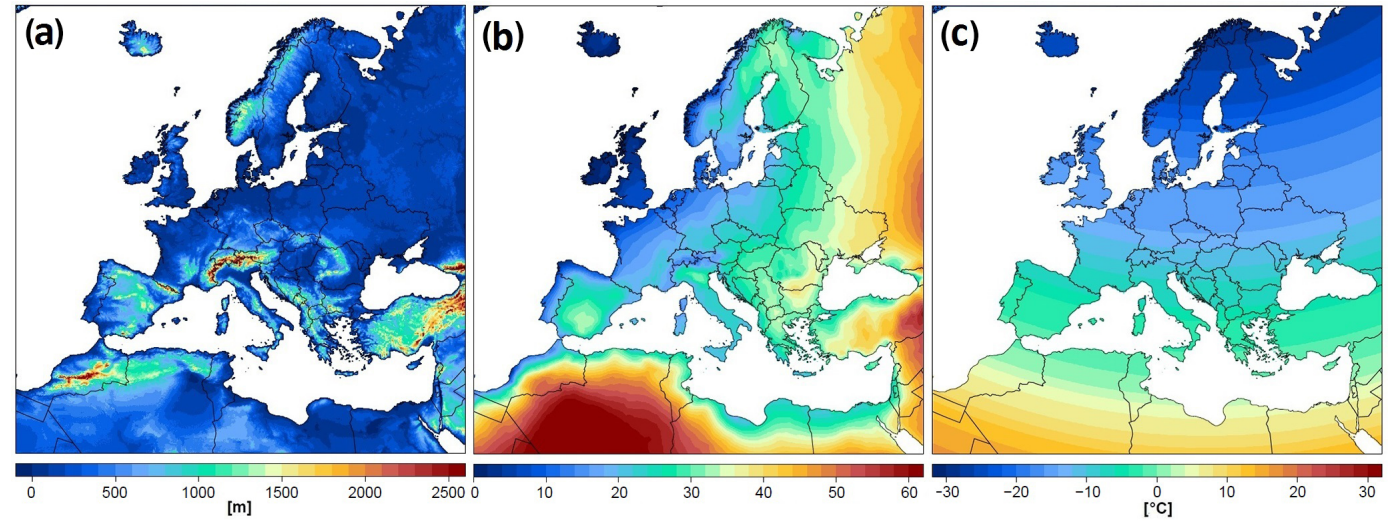

Figure 6. Predictor fields of altitude (a), continentality (b) and zonal monthly mean temperature (c; here January, based on climatology 1961-1990).

2005) is applied (see corresponding fields in Fig. 6).

$$
\begin{aligned}
T(x)= & k_{0}+k_{1} \times \operatorname{alt}(x)+k_{2} \times \operatorname{con}(x)+k_{3} \times \operatorname{zon}(x) \\
& +\operatorname{res}(x)
\end{aligned}
$$

where $T(x)$ is temperature at station $x$, alt is altitude, con is continentality index, zon is zonal mean temperature and res is residuum. These so-called predictor fields explain a major part of the spatial variation of monthly temperatures (Krähenmann et al., 2011). In order to receive a regionspecific regression model, usually only data from stations within the core region (weight one; compare Fig. 5) are used to calculate the regression coefficients. Due to the relatively sparse data density in regions 1,3 and 7, station data from the overlap areas are also considered here.

Among the three predictors, altitude is the most crucial because temperature typically strongly depends on it and altitude changes in space occur on very small scales. Thus, linear regression against altitude can substantially improve the final interpolation results in regions with pronounced orographic characteristics. In a new setup applied in this work the dependency of monthly temperature from altitude is determined first and independently from the two other predictors on the basis of station data from mountainous areas. This strategy was chosen because height coefficients derived from the standard multiple regression are potentially affected by strong horizontal temperature contrasts. For example, if mountain stations are concentrated in a part of a region with relatively low temperatures, lapse rates tend to be overestimated in the previous setup. Implausible lapse rates were diagnosed under such conditions, especially for the Scandinavian region. We could solve this issue with an independent regression step on altitude based on station data from valleys and mountains in relatively close distance. The orange dots in Fig. 5 mark stations used for this initial regression step. Depending on the region, different criteria (minimum distance to coast, minimum altitude and regional weight) are applied to receive representative subsets. Due to the absence of station data suitable for this approach in regions 3 and 7, the regression coefficients for altitude determined in regions 2 and 6 , respectively, are used here.

A second modification in the altitude regression setup was implemented in this work. Also daily temperature-altitude dependencies are estimated using the same strategy as above. In this way variations from day to day, which occur especially in winter, can be considered.

For 2010 we compared the height coefficients according to the previous setup (setup 1, involving all predictors and station data) and the new setup (setup 2, separate regression with subsets). Reference lapse rates were calculated using the regional averages of representative data pairs from mountains (highest available station in a target area, at least $700 \mathrm{~m}$ a.s.l.) and nearby valleys. The data of up to two suitable valley stations (at least $400 \mathrm{~m}$ below height of mountain station) were used for each mountain station to receive a robust average. A minimum distance of $0.8^{\circ}(\approx 90 \mathrm{~km})$ between adjacent reference mountain stations was chosen to avoid clusters in mountain areas with high station coverage. In Fig. 7 a comparison of the different setups is shown for region 2 and parameter $T_{\text {avg. The outcomes from the two setups clearly differ for }}$ the winter months (Fig. 7b). Compared to the monthly reference lapse rates (green curve) a clear overestimation of more

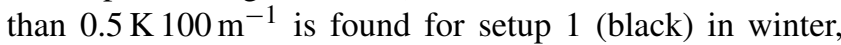
while for setup 2 (orange) a good agreement with the refer-

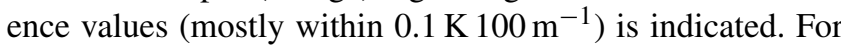
comparison, setup 1 was also used in a single regression on altitude (grey). As expected, this approach yields more unrealistic lapse rates. It is apparent that the impact of relatively warm coastal waters in winter, especially at the Norwegian coastline (with many data points), leads to the calculation of overly pronounced vertical temperature gradients. The involvement of continentality (containing the effect of temperature moderation by the oceans) in the multiple regression approach is not capable to fully balance this effect in Scandinavian winter. 

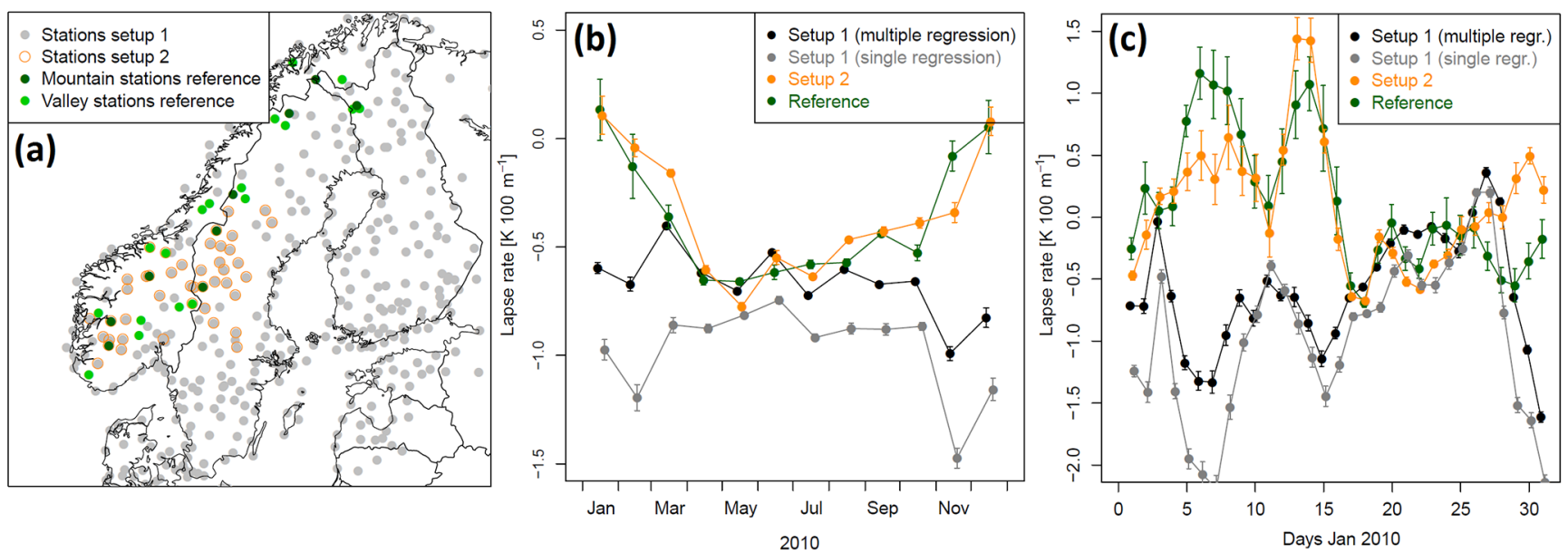

Figure 7. Comparison of regression setups used to estimate temperature lapse rates for mean temperature in Scandinavia. (a) Stations used in different setups; (b) monthly lapse rates in 2010; (c) daily lapse rates for January 2010.

In comparison to the daily reference lapse rates (Fig. 7c, for January 2010), considerable deviations were found for both setups, with mean absolute deviations of 0.9 and

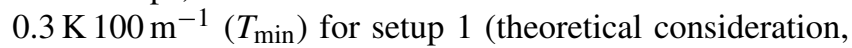
since no daily regression implemented in the previous work) and 2, respectively. Nevertheless, a clear improvement is achieved with the new setup in this problematic region. The periods of significant temperature inversions, indicated by the reference stations in the first half of the month, are not captured by the regression according to setup 1 . However, it should be noted that none of the simple approaches shown here sufficiently describe the spatial variation of temperature in mountain regions during winter (see discussion in Sect. 7). Apart from this problematic region 2, no significant differences in the results according to the two setups occurred in the tested months. In those regions absolute deviations from

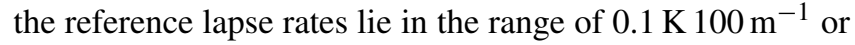
below for monthly and daily data.

Latitudinal changes of the solar radiation as well as landsea distribution and atmospheric dynamics (preferentially leading to a zonal air mass exchange) affect the predictor parameter of the long-term zonal monthly mean temperature. Continentality reflects the buffering effect of the oceans on annual temperature changes. In contrast to altitude, these two predictor fields exhibit moderate spatial changes. Their potential for improving the interpolation is thus important in regions with a low observation density, e.g., in North Africa.

Another modification compared to Krähenmann et al. (2011) is the use of station monthly means instead of climatological monthly values as input data for the monthly temperature analysis. In our work monthly hindcast periods are considered instead of current days (as in Krähenmann et al., 2011); therefore the values of the entire month are available. By using current monthly means as basis for the linear regression a potentially better adjustment of the regres-
Table 1. Whole-domain averages of spatial variance explained by single predictors $(\%)$ for monthly mean temperature and 4 tested months. In the bottom row the results for the multiple regression model involving all predictors are given.

\begin{tabular}{lrrrr}
\hline Predictor & Jan 2001 & Jan 2010 & Jul 2001 & Jul 2010 \\
\hline Altitude & 40 & 23 & 38 & 41 \\
Continentality & 28 & 33 & 52 & 53 \\
Zonal mean & 30 & 37 & 41 & 25 \\
temperature & & & & \\
\hline All & 85 & 84 & 84 & 83 \\
\hline
\end{tabular}

sion model to the mean weather conditions observed in this month is achieved and the amplitude of the regression residuals is reduced.

In Table 1 the predictive skills of the three predictors for monthly mean temperature are shown. Listed are the relative explained variances in a regression with single predictors. The corresponding results for the multiple regression model is shown in the last row. All three parameters show a high capacity to predict $T_{\text {avg }}$ on a monthly basis. Overall, more than $80 \%$ of the spatial variance can be explained by the three predictors.

\subsection{Monthly and daily kriging}

The monthly regression residuals (observations minus values according to regression model) are interpolated on a $0.011^{\circ} \times$ $0.011^{\circ}(\approx 1.25 \mathrm{~km})$ rotated basic grid (virtual North Pole at $39.25^{\circ} \mathrm{N}, 162^{\circ} \mathrm{W}$ ) using simple kriging. Simple kriging is the least complex kriging algorithm (see, for example, Stahl et al., 2006, for a comparison of the different algorithms). It requires a normal distribution of the data and thus an absence of spatial trends of the mean. This assumption is ful- 
filled, provided that most of the systematic variance has been removed by the regression step. However, a normal-score transformation (following Deutsch and Journel, 1998, attaining a standard normal distribution) is applied to the residuals prior to the interpolation. After interpolation and back transformation, block averaging is used to calculate the data on the final rotated target grid of $0.044^{\circ} \times 0.044^{\circ}(\approx 5 \mathrm{~km})$. The sum of regression field and monthly residual field results in the monthly temperature field.

In the final step the differences between daily and monthly temperatures are interpolated following the same concept as above. Before this daily interpolation all daily anomalies are height-normalized using the daily regression coefficients (correcting deviations from monthly mean) for the temperature-altitude relationship determined in step one. The daily temperature field is eventually calculated as the sum of monthly temperatures, daily height-normalized residuals and the reversal of the height normalization.

An important aspect in the interpolation using kriging is the adjustment of the kriging parameters (for details see, for example, Deutsch and Journel, 1998). These parameters estimate the change of correlation between nearby stations with distance. The related function considered in kriging is the semivariance $\gamma$, describing half the variance between all pairs of data points $Z\left(x_{i}\right), Z\left(x_{i}+h\right)$ at a certain distance, $h$, to each other.

$\gamma(h)=\frac{1}{2 N(h)} \sum_{i=1}^{N(h)}\left\{Z\left(x_{i}\right)-Z\left(x_{i}+h\right)\right\}^{2}$

The corresponding graph, illustrated in Fig. 8, is called a variogram. The variogram parameters are sill (the maximum semivariance observed in far distance from the origin), nugget (minimum semivariance observed at the origin) and range (the distance at which the semivariance levels off at the maximum). Stations outside the range are not expected to carry relevant information for a target point at the origin. The nugget, taking on values between zero and the sill, defines the noise of the data at the origin. Thus, it sets a basic uncertainty of all final gridded data in the considered region. This nugget effect can be understood as a result of measurement error and fluctuations below the spatial scale resolved by the stations. Different functions can be used to describe the change of the semivariance with distance. Here, a spherical model is assumed, following Krähenmann et al. (2011).

Several strategies of fitting the variogram function to the station data in each region were tested in this work. First, a "null" variogram is defined based on experiments with data from 4 example months (January 2001, July 2001, January 2010, July 2010). Using cross validation, thus leaving out subsequently one data point and reproducing it based on the information from the remaining stations, different combinations of the three parameters are tested. The parameter values performing best, define the "null" variogram. An automated function for fitting variograms (Pebesma, 2004) is

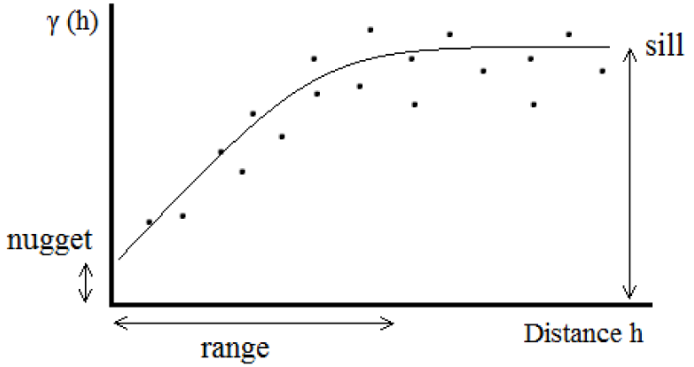

Figure 8. Idealized variogram with the parameters nugget, sill and range (after Deutsch and Journel, 1998). See description in the text.

afterwards used to further optimize the variogram. Our tests showed that, on average, both the "null" variogram based on cross-validation results and the variogram based on the automated fitting perform equally well, but in rare cases the automated fitting algorithm fails to determine reasonable results due to a missing convergence in the fitting based on least squares (Pebesma, 2004). In the final setup we decided to use the "null" variogram as a robust basis and allow a slight adjustment of the parameters in cases where clear differences between the two models occur. The parameters nugget and range of this "null" variogram are listed in Table 3 for monthly and daily kriging of $T_{\text {avg }}$ in the seven regions. The nugget values can be interpreted as percentage of the background noise measured at the origin. For $T_{\text {avg }}$ relatively low nugget-to-sill ratios between 0.1 and 0.3 were determined. Thus, a relatively strong spatial dependence of the residual fields is indicated. The ranges, within which station data are correlated, are between 5 and $8^{\circ}$ on the rotated grid $(\approx 550$ to $900 \mathrm{~km}$ ).

\section{Interpolation procedure wind speed}

For the interpolation of daily wind speed a new method based on the concept used for temperature was developed. Different predictor fields correlated with wind speed were tested and chosen. Again, the seven regions displayed in Fig. 5 are applied. In addition, a new region for the Alps is introduced (Fig. 9). The motivation for this new region will be outlined in the next section.

\subsection{Regression}

After testing a variety of potential predictor fields, four parameters were chosen for the linear regression (see Fig. 10).

$$
\begin{aligned}
V(x)= & k_{0}+k_{1} \times \exp (x)^{0.5}+k_{2} \times \ln (\operatorname{coa}(x)+1) \\
& +k_{3} \times \ln \frac{10}{z_{0}(x)}+k_{4} \times \operatorname{era}(x)+\operatorname{res}(x),
\end{aligned}
$$

where $V(x)$ is wind speed at station $x$, exp is exposure or relative altitude, coa is coastal distance, $z_{0}$ is surface roughness length, era is ERA-Interim $850 \mathrm{hPa}$ reanalysis wind speed 


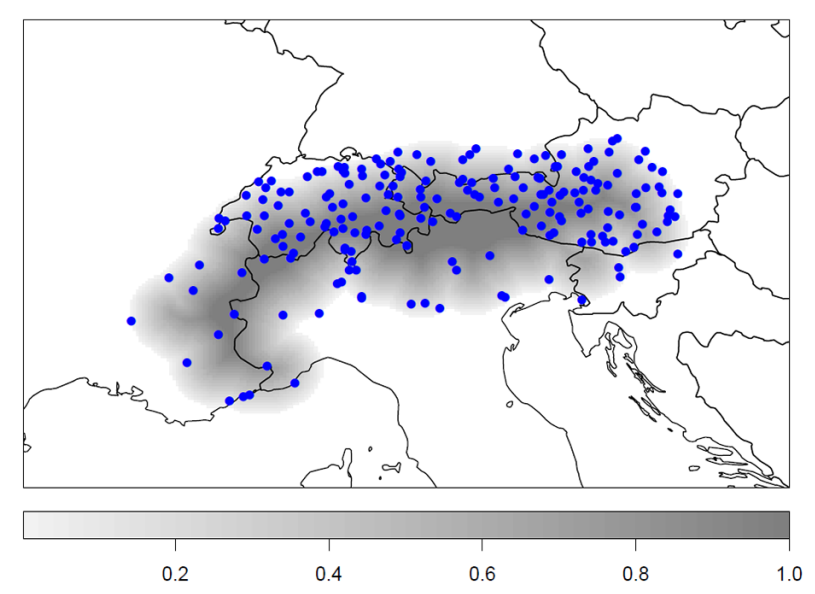

Figure 9. Alpine region (denoted region 8 in the following) introduced for the interpolation of wind speed.

and res is residuum. The use of relative altitude (in the following the term exposure will be used as a synonym) was motivated by Walter et al. (2006), who found good correlations with $10 \mathrm{~m}$ wind speed in Germany for altitude at a given point transformed to exposure by dividing it with the mean altitude of the surrounding area of $10 \mathrm{~km} \times 10 \mathrm{~km}$. Here, we calculated corresponding fields on a $1 \mathrm{~km}$ grid using elevation data (same sources as in Sect. 3.1) and applying a radius of $5 \mathrm{~km}$ for the determination of surrounding mean altitude. Block averages were calculated to obtain data for the final target grid of $5 \mathrm{~km} \times 5 \mathrm{~km}$. For station data the exact altitude reported is used in comparison with the $1 \mathrm{~km}$ grid of mean altitudes described above. We tested different functions to find the most suitable relationship between exposure and $10 \mathrm{~m}$ wind speed (e.g., linear, logarithmic and different power functions). On average, exposure to the power of 0.5 (equal to square root) showed highest correlations.

Coastal distance is also of high relevance for the mean wind speed in $10 \mathrm{~m}$ since the very low roughness across the sea surface, related to very low friction, leads to typically stronger winds in the vicinity of coastlines. Our tests showed the best performance when using the logarithm of the coastal distance in the form $\ln (\mathrm{coa}+1)$ and defining maximum coastal distances (higher values are reduced to that constant) between 20 and $100 \mathrm{~km}$. This maximum distance is chosen individually for each month and region on the basis of the lowest root mean square error (RMSE) for a regression on coastal distance.

Surface roughness describes the deviations of a surface from an ideal smooth form. On the Earth's surface obstacles such as bushes, trees or buildings increase the surface roughness and thus affect the movement of air. According to theory the wind speed change with distance from the surface shows the following simplified dependency (under neutral stability conditions) on the roughness length $z_{0}$ (see, for example, Holton and Hakim, 2012):

$v(z)=\frac{v_{*}}{\kappa} \times \ln \frac{z}{z_{0}}$,

where $v(z)$ is wind speed at height $z$, von Karman constant is $\kappa$ and shear velocity is $v_{*}$. Based on this equation we apply the roughness length in the regression step as $\ln \left(10 / z_{0}\right)$ because linear dependency on $v$ is expected if $v_{*}$ is assumed constant (valid in the lowest $10 \mathrm{~m}$ considered here). Here, we use roughness length data in $1 \mathrm{~km}$ resolution derived from the global land cover data set GLC2000 (Bartholome and Belward, 2005).

In addition to the three "static" predictor parameters above, the use of meteorological field data can provide valuable information for regions with low station coverage. Krähenmann and Ahrens (2013) showed that the inclusion of remote sensing data from satellite observations as a predictor in regression kriging substantially improves the gridding of surface temperature over the Iberian Peninsula. For wind speed, relevant satellite measurements of high quality are currently not available. However, air pressure fields, as the initial driving force of large-scale air movement, are well analyzed in weather models. Here, we tested ERA-Interim reanalysis data provided by the European Centre for MediumRange Weather Forecasts (ECMWF). These data are available in 6-hourly resolution on a reduced Gaussian grid with a grid point distance of approximately $80 \mathrm{~km}$, from $1979 \mathrm{un}$ til today (Dee et al., 2011) at http://apps.ecmwf.int/datasets/ data/interim-full-daily/. In order to maintain independence from the other three predictors, corresponding model fields of geopotential height as well as the direct output for wind speed in pressure levels between 850 and $700 \mathrm{hPa}$ (reflecting conditions in the nearly "free" atmosphere, undisturbed by surface impacts) were examined as predictor. Horizontal gradients derived from geopotential height and model wind speeds showed best correlations with surface station wind speed for the lowest tested level $850 \mathrm{hPa}$. This level corresponds with altitudes of around $1500 \mathrm{~m}$; thus in high mountain areas like the Alpine region the data fields intersect with the land surface and therefore are affected by surface effects. In these cases an independence from the predictor fields of exposure and roughness length is not ensured. In the $700 \mathrm{hPa}$ pressure level the influence of high mountains nearly vanishes but the correlations are generally weaker. For the final regression setup we decided to use ERA-Interim wind fields at $850 \mathrm{hPa}$. Scalar wind speeds are derived from the two vector components $u$ and $v$. Daily means are calculated in the same way as described in Sect. 2.2. For grid points above $1000 \mathrm{~m}$ reanalysis data are eliminated and afterwards re-estimated by the information of adjacent data points. Finally, daily and monthly ERA-Interim scalar wind speed data fields are interpolated to the target DecReg grid using bilinear interpolation.

Despite the filter algorithm applied for reanalysis data in regions of high altitudes, a slight dependency of reanalysis 

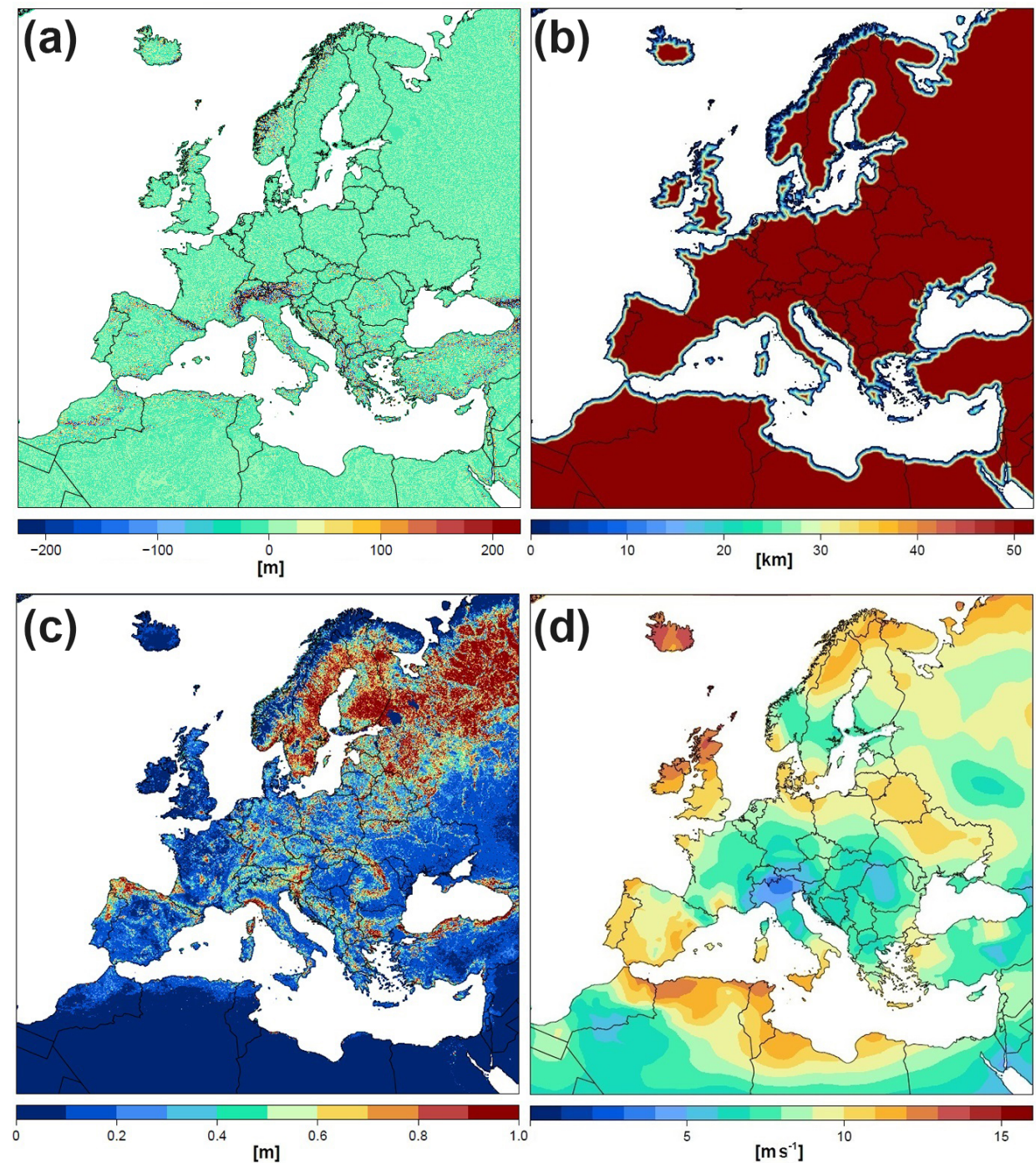

Figure 10. Predictor fields of exposure (a), coastal distance (b), roughness length (c), ERA-Interim mean scalar wind velocity in $850 \mathrm{hPa}$ (d; here January 2001).

data on exposure and roughness length remains for areas like the Alps, the Atlas Mountains, the Caucasus and parts of Turkey. Thus, for the Alps, where a good coverage with station data is available, a new region (Fig. 9) was set up, for which regression on reanalysis wind speed is omitted. All grid points above $1500 \mathrm{~m}$ in this area receive regional weight one. An overlap to adjacent regions was determined and the regional weights of the adjacent regions were adjusted. Tests indicate a slight improvement of the overall explained variance in 3 out of 4 tested months (January/July 2001/2010) for this new configuration.

The four predictors are used for the regression of the data of monthly mean wind speed. In contrast to temperature, wind speed data tend to produce a logarithmic distribution. Therefore, ratios between monthly wind data and the corresponding area mean of the related region are considered.
Table 2. Whole-domain averages of the spatial variance explained by single predictors $(\%)$ for monthly mean wind speed and 4 tested months. The bottom row shows the result for the multiple regression model involving all predictors.

\begin{tabular}{lrrrr}
\hline Predictor & Jan 2001 & Jan 2010 & Jul 2001 & Jul 2010 \\
\hline Exposure & 24 & 20 & 18 & 17 \\
Coastal distance & 28 & 35 & 27 & 28 \\
Roughness length & 23 & 28 & 22 & 24 \\
ERA-Interim $850 \mathrm{hPa}$ & 10 & 13 & 11 & 21 \\
\hline All & 58 & 58 & 51 & 53 \\
\hline
\end{tabular}

Core regions relevant for the determination of the regression coefficients in each region were defined in the same way as for temperature. For the new region 8 , weights above 0.5 de- 
Table 3. Regional variogram parameters nugget (relative to sill) and range ( ${ }^{\circ}$ rot. grid) based on experiments with temperature data $T_{\text {avg }}$ of 4 example months (January 2001, July 2001, January 2010 and July 2010). Listed are the regional averages for monthly and daily interpolation.

\begin{tabular}{|c|c|c|c|c|c|c|c|}
\hline Parameter & Region 1 & Region 2 & Region 3 & Region 4 & Region 5 & Region 6 & Region 7 \\
\hline \multicolumn{8}{|l|}{ Monthly } \\
\hline Nugget & 0.1 & 0.1 & 0.1 & 0.1 & 0.1 & 0.2 & 0.1 \\
\hline Range & 5 & 5 & 7 & 6 & 8 & 6 & 7 \\
\hline \multicolumn{8}{|l|}{ Daily } \\
\hline Nugget & 0.3 & 0.1 & 0.1 & 0.2 & 0.1 & 0.2 & 0.1 \\
\hline Range & 6 & 6 & 8 & 6 & 8 & 7 & 8 \\
\hline
\end{tabular}

Table 4. Regional variogram parameters nugget (relative to sill) and range ( ${ }^{\circ}$ rot. grid) based on experiments with wind speed data $V_{\text {avg }}$ of 4 example months (January 2001, July 2001, January 2010 and July 2010). Listed are the regional averages for monthly and daily interpolation.

\begin{tabular}{|c|c|c|c|c|c|c|c|c|}
\hline Parameter & Region 1 & Region 2 & Region 3 & Region 4 & Region 5 & Region 6 & Region 7 & Region 8 \\
\hline Monthly & & & & & & & & \\
\hline Nugget & 0.8 & 0.5 & 0.7 & 0.3 & 0.6 & 0.6 & 0.8 & 0.3 \\
\hline Range & 2 & 4 & 3 & 5 & 6 & 2 & 2 & 4 \\
\hline Daily & & & & & & & & \\
\hline Nugget & 0.7 & 0.4 & 0.5 & 0.3 & 0.4 & 0.4 & 0.5 & 0.7 \\
\hline Range & 2 & 4 & 5 & 3 & 4 & 3 & 4 & 1 \\
\hline
\end{tabular}

fine its core area. The test results of the monthly regression for the 4 example months are listed in Table 2. Each of the parameters explain a considerable part of the spatial variance of $V_{\text {avg }}$. Overall, around $55 \%$ of the variance of the monthly mean is captured by the regression model. The values are somewhat lower than those obtained for temperature regression. This can partly be explained by the high dependency of wind speed on local characteristics not captured by the regression.

Also, linear regression on a daily basis was tested, focusing especially on the predictive skill of the daily ERAInterim reanalysis data. Thereby we found good correlations between ERA-Interim and the daily observations. On average, $31 \%$ of daily variance could be explained by ERAInterim over 4 tested months (same as above). Thus, an additional regression step on a daily basis is applied using daily anomalies of ERA-Interim $850 \mathrm{hPa}$ wind data from the corresponding monthly means.

\subsection{Monthly and daily kriging}

Following the same scheme as described for temperature, the normal-score transformed residuals of the monthly regression are interpolated using simple kriging. Again, a "null" variogram optimized on the basis of cross-validation experiments for 4 tested months (same as above) was determined for monthly and daily means in each region. The results are shown in Table 4. Compared to temperature the nuggets are somewhat larger and the ranges smaller. Thus, for wind speed the noise of the regression residuals at the origin of each tar- get grid point is, on average, relatively large and the interpolation uncertainty relatively high. Regional signals vanish at distances of 2 to $6^{\circ}(\approx 220$ to $670 \mathrm{~km})$.

After normal-score back transformation the gridded monthly residuals are added to the gridded regression values and multiplied by the absolute mean wind speed of the considered region (correcting the normalization applied before regression) to obtain the monthly field of $V_{\text {avg }}$.

In the daily kriging step the daily anomalies with respect to the monthly mean at each station are interpolated. Here, ratios instead of absolute deviations are considered, respecting the characteristics of wind speed distribution. As noted in Sect. 4.1, an additional regression with regard to daily ERAInterim $850 \mathrm{hPa}$ wind speed is performed prior to the interpolation. The final daily wind field is calculated involving the daily regression field, the back transformed daily anomaly ratios and the monthly field of $V_{\text {avg }}$.

\subsection{Uncertainties of interpolation}

For each of the three interpolation steps uncertainty estimates are recorded. For the two kriging steps the kriging variance is used as a measure of uncertainty. Kriging variance is known to lack precision on a local scale, since local variation of the data is not considered in the estimation of uncertainty. More sophisticated approaches were suggested by Deutsch and Journel (1998) and Yamamoto (2000). However, due to the enormous increase in computing time, as observed in tests using the approach by Yamamoto (2000), we rely on the easily available kriging variance in this work. Regression 

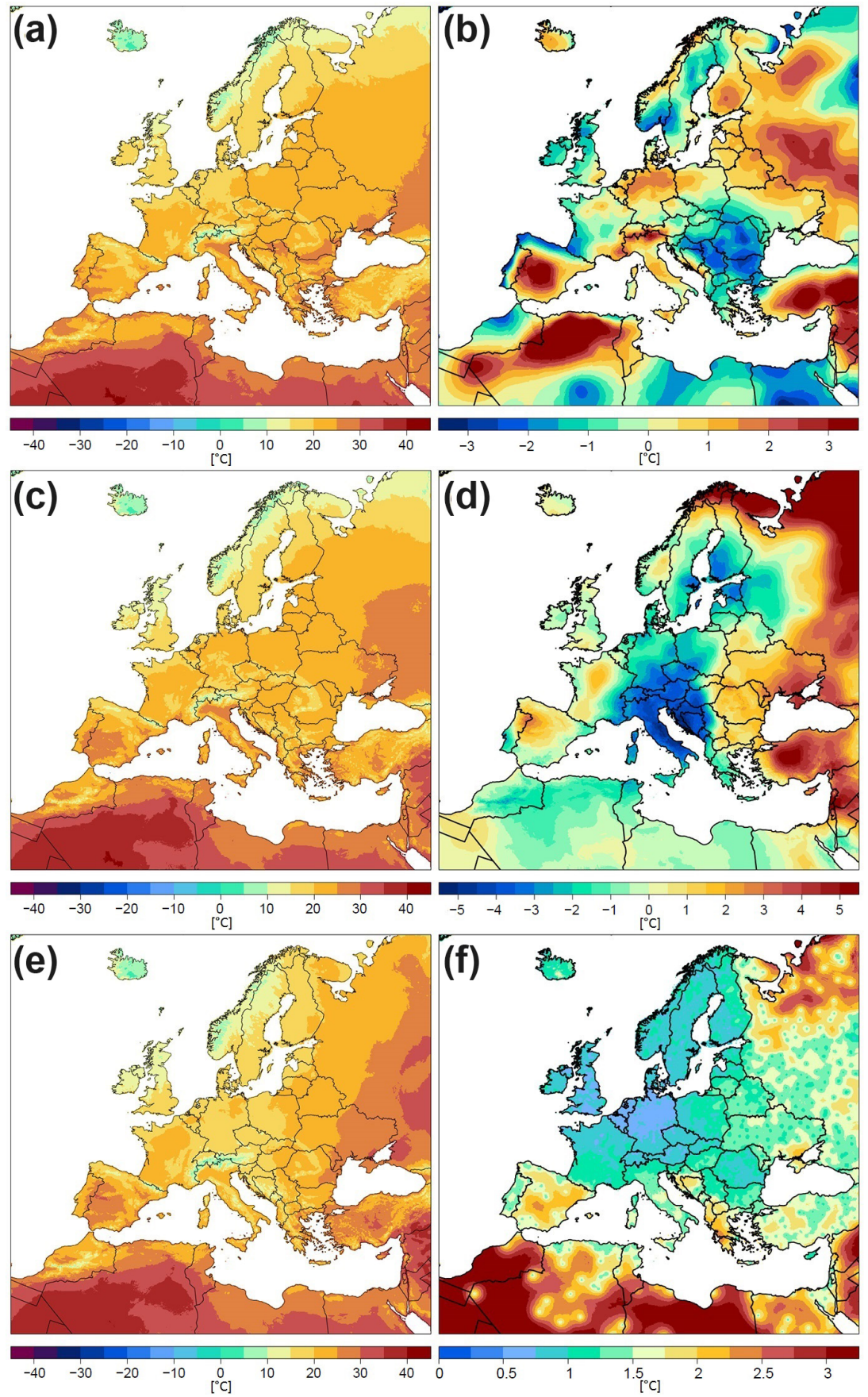

Figure 11. Steps in the interpolation of daily mean temperature for 31 July 2010. (a) Monthly regression field; (b) monthly regression residuals; (c) monthly mean temperature; (d) daily anomaly with respect to monthly mean temperature; (e) daily mean temperature; (f) interquartile range of daily mean temperature.

and kriging errors (kriging variance and semivariance at the origin) are combined according to error propagation to determine total uncertainties. Finally, interquartile ranges (IQRs; range between 0.25 and 0.75 quantile, thus containing $50 \%$ of the data) are recorded for all monthly and daily gridded data sets.
Furthermore, cross validation for all data within the example years 2001 and 2010 is applied to receive error estimates for all station coordinates. In Sect. 5.5 a comparison between cross-validation results and the uncertainty estimates based on kriging variance is presented. 

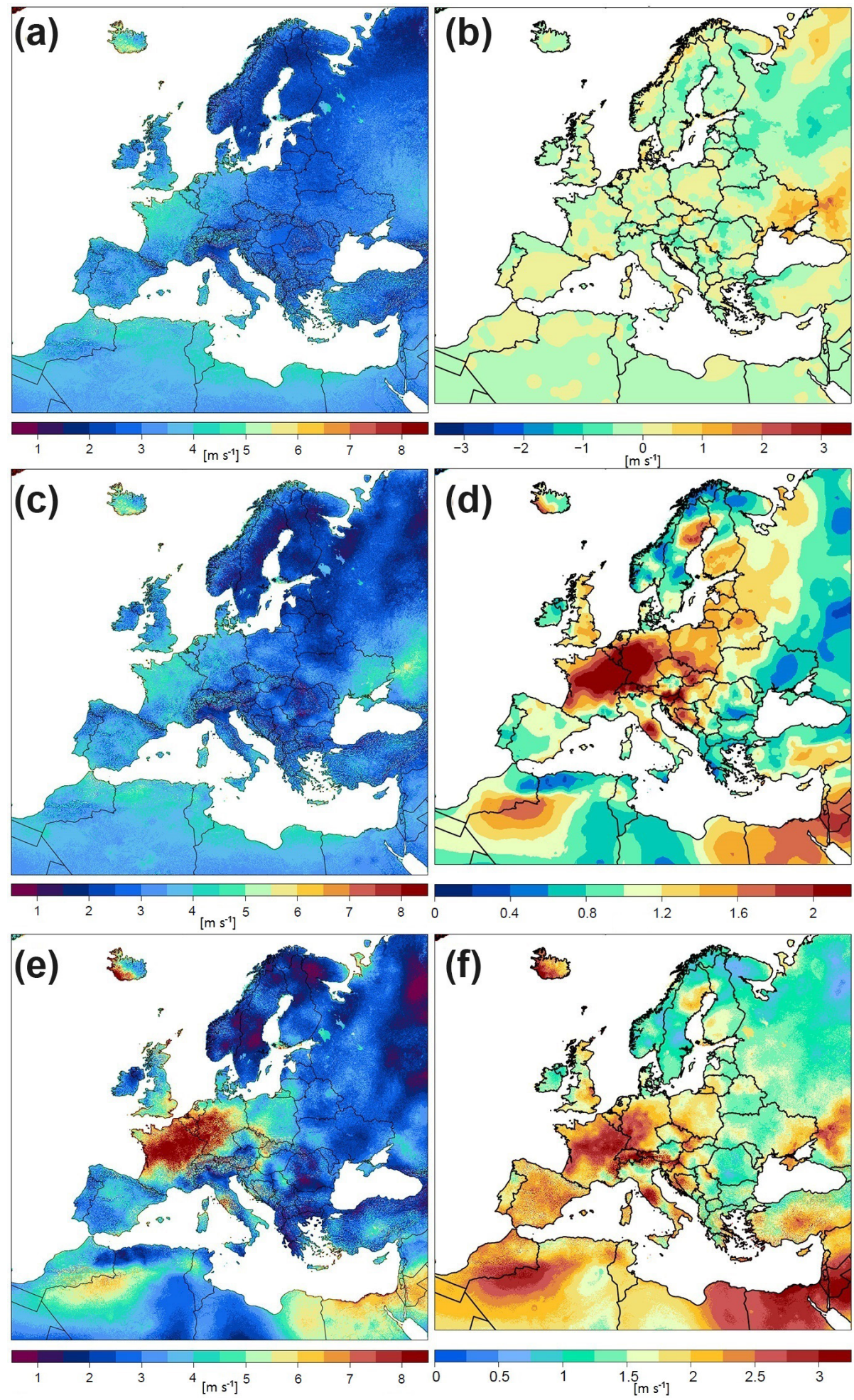

Figure 12. Steps in the interpolation of daily mean wind speed for 28 February 2010. (a) Monthly regression field; (b) monthly regression residuals; (c) monthly mean wind speed; (d) ratio daily to monthly mean wind speed; (e) daily mean wind speed; (f) interquartile range of daily mean wind speed. 

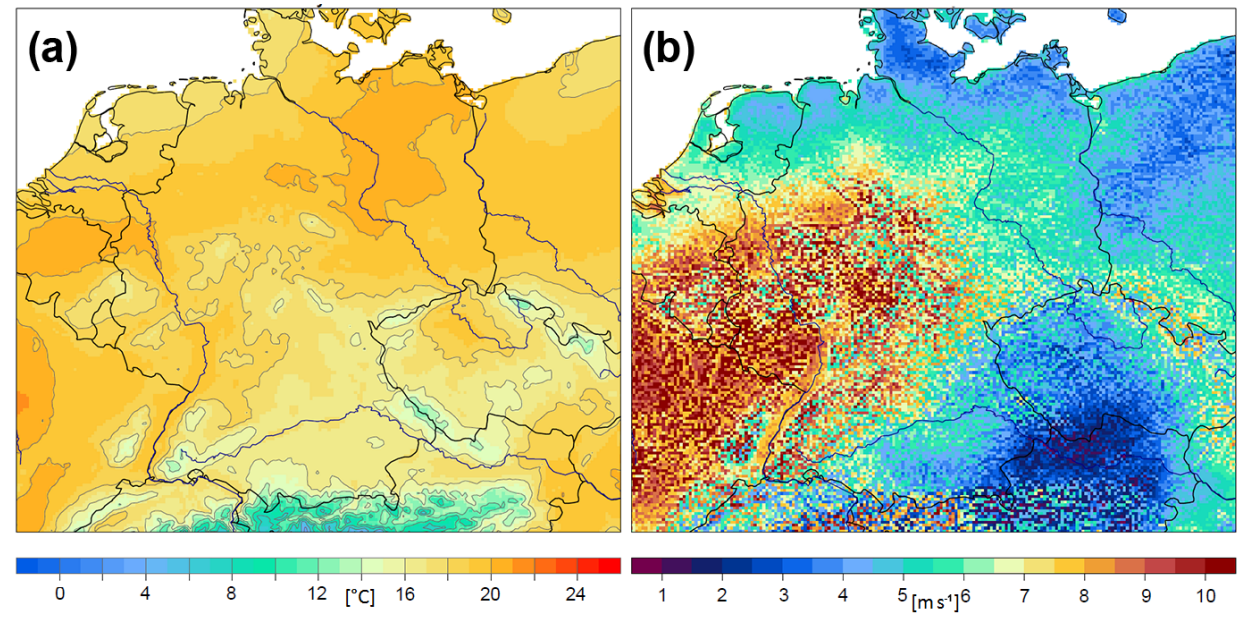

Figure 13. Detailed image of central Europe for (a) daily mean temperature (31 July 2010) and (b) daily mean wind speed (28 February 2010).

\section{Results and discussion}

\subsection{Example outputs}

In Fig. 11 the basic interpolation steps for the generation of the gridded field of daily mean temperature are illustrated. The monthly field (Fig. 11c) is calculated as the sum of regression field (Fig. 11a) and the interpolated residuals (Fig. 11b). The interpolated daily anomalies (Fig. 11d) from the monthly data are used to determine the final grid of daily mean temperature (e, here for 31 July 2010). The uncertainties of the daily data are characterized by the IQR fields shown in Fig. 11f. In the central and northwestern part of the domain the quality is indicated to be very high, with IQR around $1.0 \mathrm{~K}$. In other regions, where the coverage of station measurements is lower (compare Fig. 1), higher IQRs partly exceeding $3 \mathrm{~K}$ are recorded.

Corresponding results for daily mean wind speed on 28 February 2010 are shown in Fig. 12. Instead of absolute daily anomalies here the ratios to the monthly means are interpolated (Fig. 12d). Note that the intermediate step of daily regression on $850 \mathrm{hPa}$ reanalysis winds (as for daily regression on altitude in temperature scheme in Fig. 11) is not displayed here. The uncertainties of the final daily wind speed data are relatively high in areas of high wind speeds. In contrast to temperature, the dependency on the station density is lower because a considerable amount of the spatial variability is not captured by station measurements and predictor fields (see discussion on the nugget effect in Sect. 3.2). To illustrate the small-scale characteristics of the interpolation products, the two example outputs for daily mean temperature and daily mean wind speed are displayed for central Europe in Fig. 13.

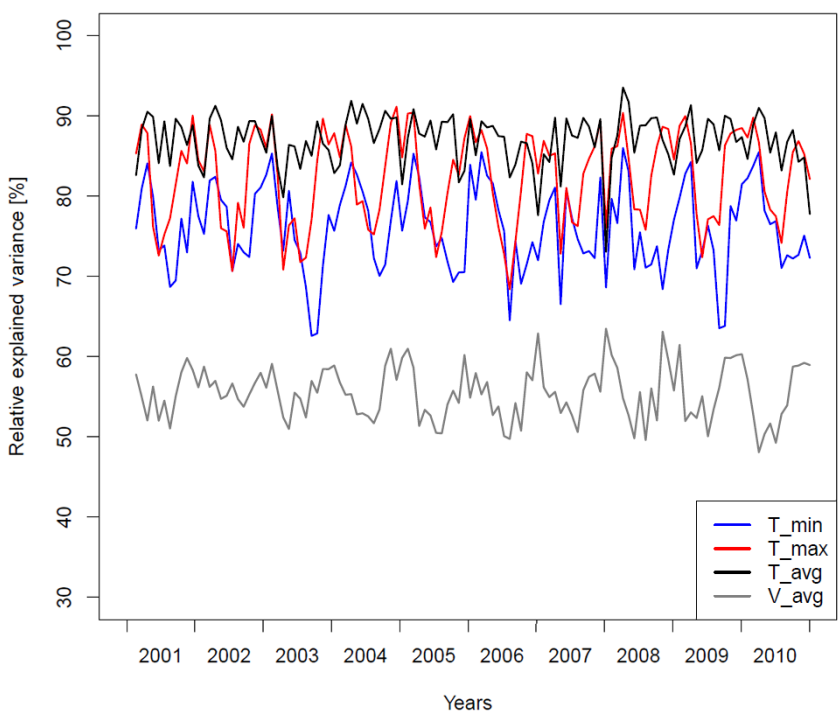

Figure 14. Relative explained variance for monthly mean wind speed and for the monthly mean of the three temperature parameters (see color code) for 2001-2010.

\subsection{Regression}

In the following the results of the monthly regression analysis for the full decade 2001-2010 are presented for wind speed and for the three temperature parameters. In Fig. 14 the spatial variance explained by the predictors $(\mathrm{EV})$ is displayed for the entire decade of 2001-2010. Highest values of up to around $90 \%$ are reached for $T_{\text {avg. }}$. For $T_{\min }$ and $T_{\max }$ the EV values fluctuate at slightly lower levels of around $80 \%$. Concerning wind speed, the EV values are considerably lower (around 55\%). Nevertheless, taking into account its high degree of small-scale variability, the EV values realized here for wind speed represent a promising result. Over 

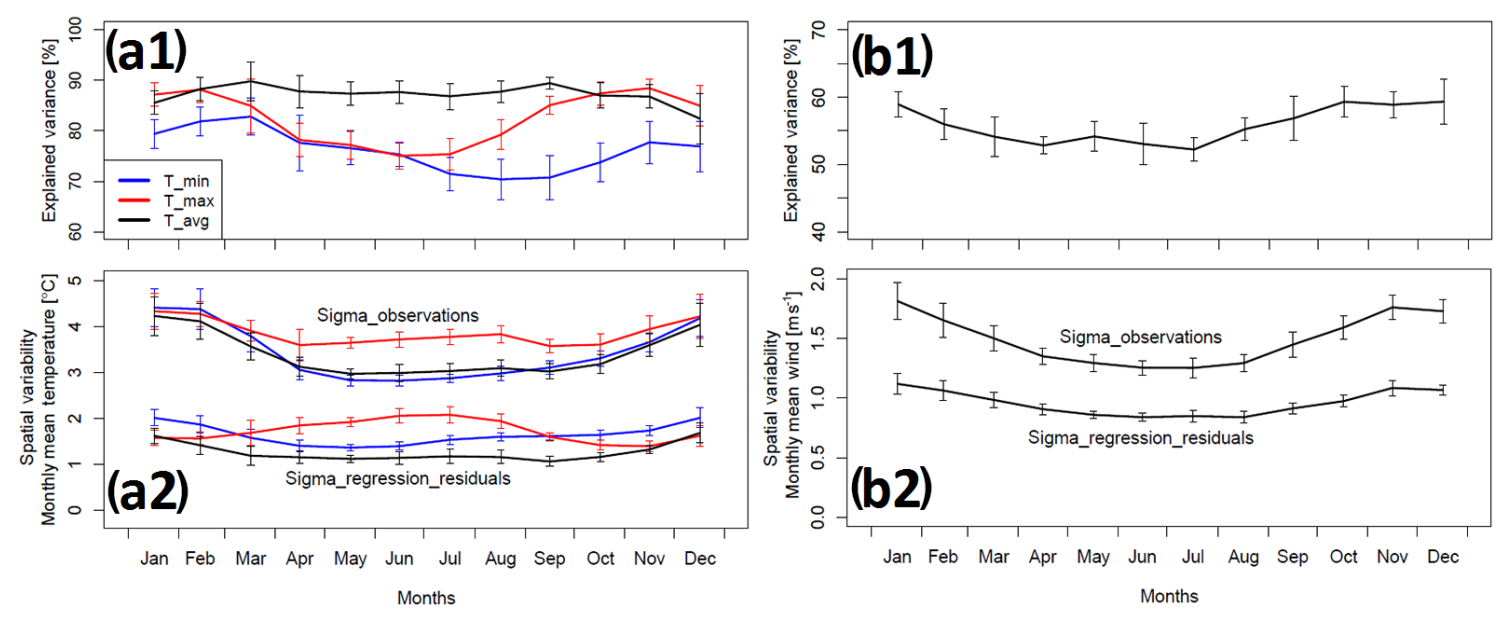

Figure 15. Annual cycles (means 2001-2010) of relative explained variance for monthly mean temperature (a1, see color code of the parameters) and wind speed (b1) and corresponding spatial variability ( $1 \sigma)$ before and after regression (a2, b2). All data are given as means over all regions. The error bars display the temporal standard deviation over the 10 years.

the 10 years no visible trend as a result of the trend in the number of station data is found. However, the curves indicate annual cycles caused by seasonal changes in spatial variance and/or the predictive capacity of the predictor fields.

This aspect is investigated more closely in Fig. 15. Here, annual cycles based on the statistics over the 10 years for EV and the spatial variability (standard deviation) of the raw data and the regression residuals are displayed. For temperature (Fig. 15a1, a2; see color code of the three parameters) a generally higher spatial variability during winter is observed. For $T_{\max }$ an additional summer maximum is visible, the unexplained variance even peaks in summer for this parameter. However, the high predictive capacity of the three predictors leads to a strong reduction of the residual variability for temperature.

For wind speed (Fig. 15b1, b2) similar annual cycles with winter maxima are indicated. After regression the remaining spatial variance is considerably reduced.

\subsection{Interpolation - cross validation}

For two years, 2001 and 2010, the quality of the final interpolation product is evaluated by applying "leave one out" cross validation (as defined in Sect. 3.2). Combining the crossvalidation results for the monthly and the daily interpolation yields uncertainty estimates of the gridded data near each target station. Figure 16 displays corresponding results for January 2010 (Fig. 16a1, a2) and July 2010 (Fig. 16b1, b2) for daily mean temperature. Figure 16a1 and b1 show the mean absolute error of the 31 daily values at each station (see color code). The corresponding statistics over all days and stations are summarized in Fig. 16a2 and b2.

The RMSE is $1.68 \mathrm{~K}$ in January and $1.00 \mathrm{~K}$ in July. Thus, interpolation of mean temperature is, on average, considerably more accurate for the summer month considered here.
This finding is consistent with the relatively low variability of the regression residuals in summer (compare with Fig. 15). However, the regional distribution of the errors exhibits clear spatial differences: while in the north a tendency towards higher errors in winter is found, the southern regions reveal highest errors mainly in summer. This can possibly be explained by the relatively low predictive capacity for night temperatures during cold winter periods (especially in complex terrain; e.g., cold air pools) and for day temperatures under high insolation in summer (affected by clouds, convective precipitation, coastal waters). This assumption is supported by the annual cycles of the regression residuals in Fig. 15. Especially during periods of temperature inversion in winter the simple linear regression approach on altitude is not capable of reproducing the spatial temperature variation in mountainous regions satisfactorily (e.g., Frei, 2013). A discussion on this aspect will follow in Sect. 7. Overall, very accurate interpolation results are found in regions with a high observation density and low topographic complexity.

Hofstra et al. (2008) have compared the skill scores for daily temperature interpolation results based on different methods. The RMSEs calculated in our study for the same domain are in the same range as found for the bestperforming methods tested in Hofstra et al. (2008). For instance, the three-dimensional thin-plate splines method used (in combination with external drift kriging) for the E-OBS temperature grid record (Haylock et al., 2008) showed RMSEs of 1.12 and $1.40 \mathrm{~K}$ for summer and winter half, respectively. A direct comparison between E-OBS grid data and the temperature data of our study is presented in Sect. 5.4.

Figure 17 shows the results of the cross validation for daily mean wind speed. Again, January (Fig. 17a1, a2) and July 2010 (Fig. 17b1, b2) were investigated. As observed for daily mean temperature, also daily mean wind speed shows a somewhat larger spread in the error distribution in January 

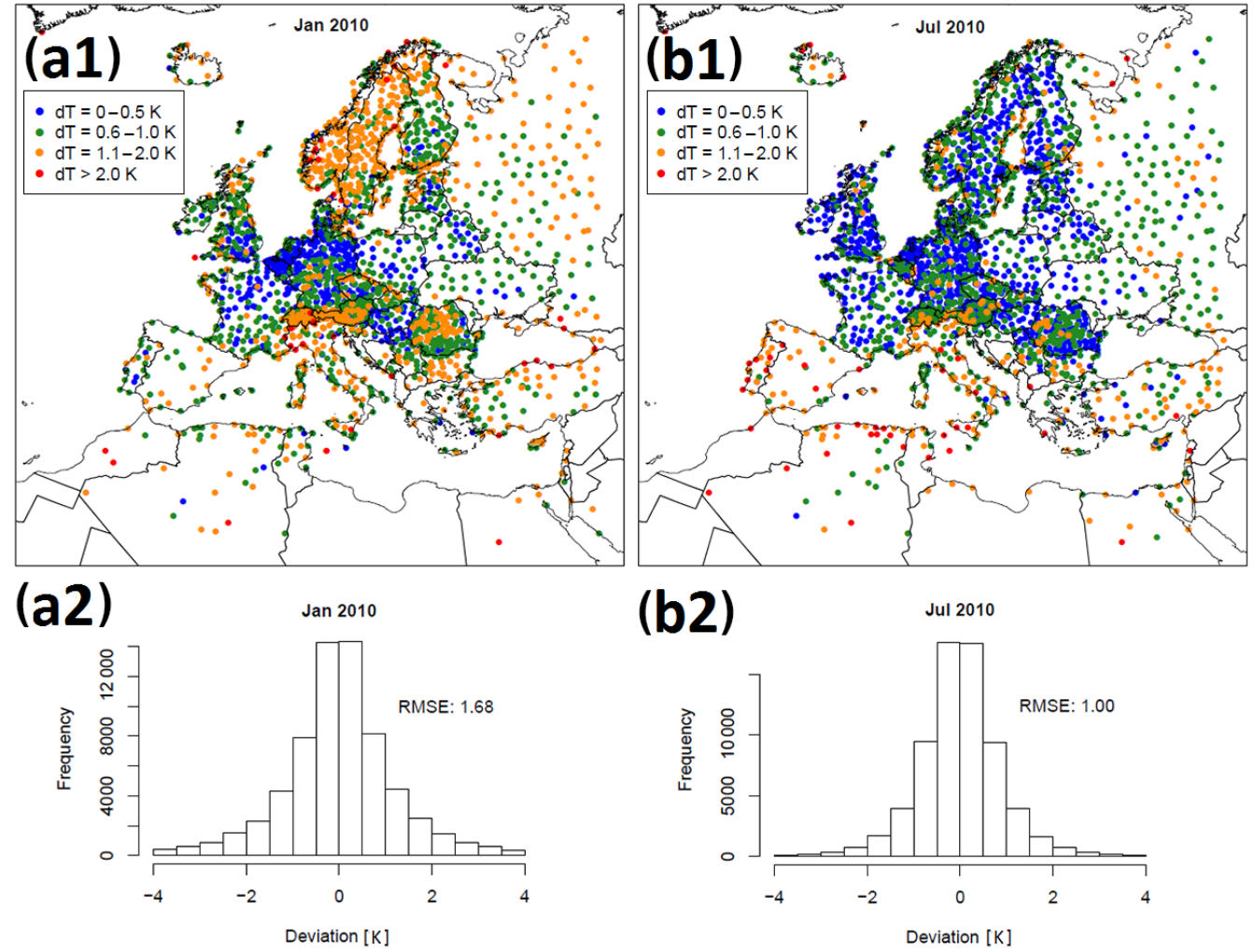

Figure 16. Cross-validation results for daily mean temperature data in January 2010 (a1, a2) and July 2010 (b1, b2). The color code indicates the monthly mean of the daily absolute deviations. The histograms contain the full statistics of deviations over all days and stations.

(RMSE of 1.42 compared to $1.06 \mathrm{~m} \mathrm{~s}^{-1}$ in July). However, seasonal differences can partly be attributed to the higher mean wind speeds occurring in January 2010 (mean over all stations: 3.37 compared to $3.05 \mathrm{~m} \mathrm{~s}^{-1}$ in July). Relatively high absolute errors are found for stations in coastal and mountainous areas and thus at sites with high wind speeds. Nevertheless, the discrepancies diagnosed for highly exposed stations on the top of mountains typically show a systematic underestimation compared to the observed values (not illustrated in the figure, as absolute deviations are given). Thus, systematic variance caused by the topography is not satisfactorily explained by the regression for areas of very high exposure.

In Fig. 18 the outcomes of cross validation are summarized for the entire annual cycles of 2001 and 2010 for the four parameters: (a) $V_{\text {avg }}$, (b) $T_{\text {avg }}$, (c) $T_{\min }$ and (d) $T_{\max }$. The black and the blue curves illustrate the two cycles of daily RMSE. For comparison the time series of daily standard deviation over all station observations are displayed in brown and orange. In addition to the daily values curves of monthly means are shown. As indicated in Figs. 16 and 17, the interpolation accuracy is clearly higher during warmer seasons for the daily means of wind speed and temperature. Only for $T_{\max }$ is a tendency towards higher errors in summer indicated. The interpolation quality is generally somewhat lower for the ex- treme temperatures (see grey curves for $T_{\text {avg }}$ added to the plots $\mathrm{c}$ and $\mathrm{d}$ for comparison). The temporal averaging used to calculate daily means leads to a reduction of unexplained variance (compare with in Fig. 15a2) and thus increases the accuracy of the interpolation.

The variability curves in Fig. 18 can be interpreted as the RMSE for the simple assumption in which the mean over all station data is assigned to each station location. Thus, the difference between lower and upper curves can be understood as a measure for the skill of the interpolation method. For wind speed this skill is much lower than for temperature due to the large fraction of unexplained variance.

Besides the accuracy of the interpolation, expressed here in the global measure RMSE, its ability to preserve the observed spatial variability is also of importance. Some methods tend to smooth small-scale features (Luo et al., 2008). Regression kriging is known to preserve spatial variance well, provided that the predictors can explain a major part of the observed variance (e.g., Krähenmann et al., 2011). Here, the cross-validation results were used to assess this aspect. Figure 19 shows the time series of relative variance, defined here as the ratio of spatial variance of interpolated and observed station data, for the years 2001 and 2010 (see color code indicating the different parameters and years). For the variance of the temperature parameters a good agreement be- 

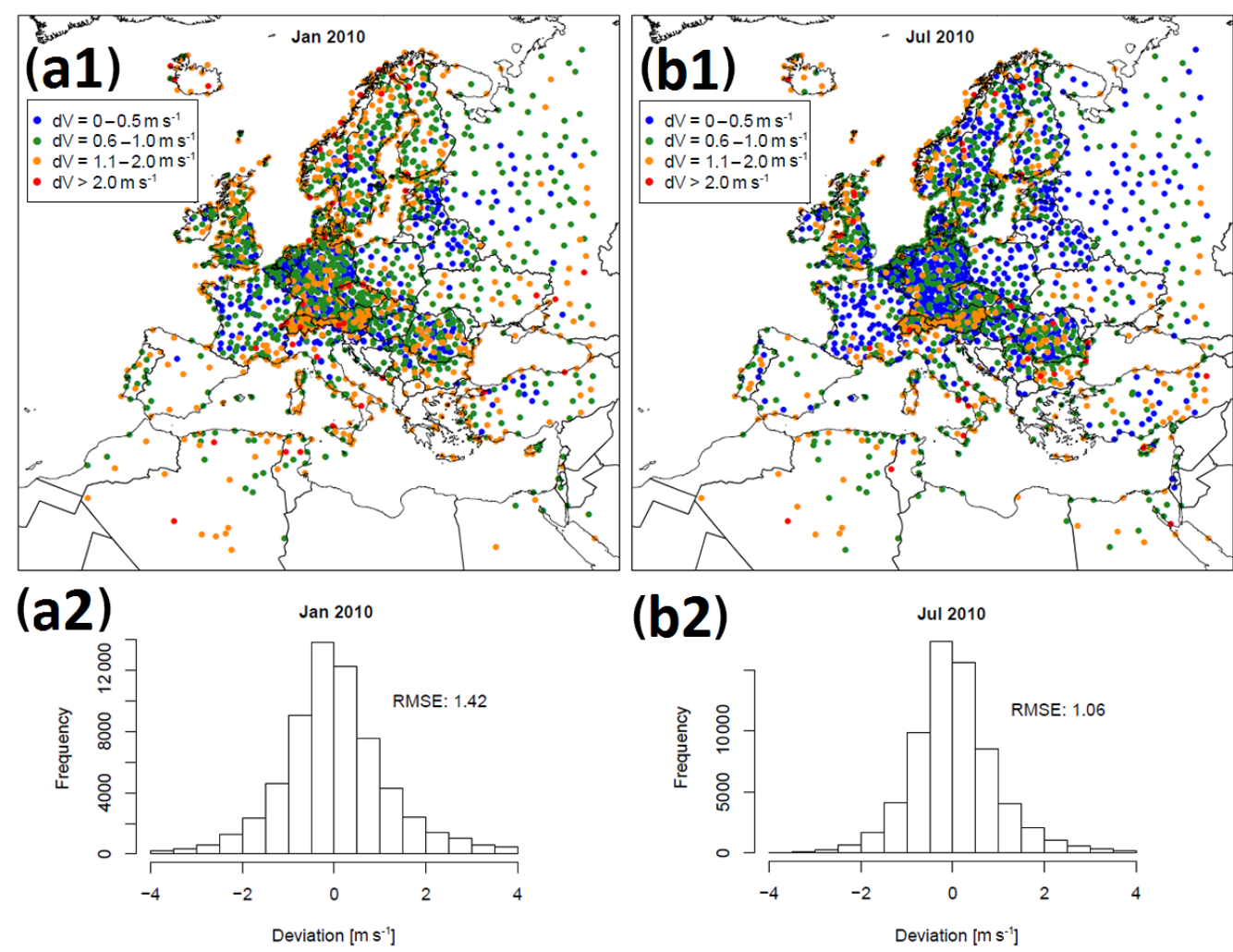

Figure 17. Cross-validation results for daily mean wind speed data January 2010 (a1, a2) and July 2010 (b1, b2). The color code indicates the monthly mean of the daily absolute deviations. The histograms contain the full statistics of deviations over all days and stations.

tween observations and interpolation results is found. Only for $T_{\min }$ are significantly underestimated variances detected during certain periods. For wind speed the relative variances fluctuate around a level slightly below 0.7 (outliers for single days in April 2001 due to very low number of station data). This low variance ratio is caused by the high degree of unexplained variance observed on very small scales (nugget effect, Sect. 4.2). The reproduction of a data value at a certain station by a weighted average of surrounding values with a large spread leads, on average, to a reduced signal at this station.

\subsection{Comparison with E-OBS grid data}

To assess the characteristic of the temperature data set in comparison with the daily E-OBS grid data (version 13.0; Haylock et al. (2008); www.ecad.eu/download/ensembles/ ensembles.php), the data fields of daily mean temperature were compared for 2010. To meet the lower resolution of EOBS $\left(0.22^{\circ}\right)$, the DecReg data were transferred to the E-OBS grid using first-order conservative remapping. For 31 January 2010 (Fig. 20) we find very similar temperature fields of $T_{\text {avg }}$ for DecReg (Fig. 20a) and E-OBS (Fig. 20b) in most parts of the domain. However, the difference field DecReg minus E-OBS (Fig. 20c) reveals major discrepancies in Russia and in mountain regions around the Mediterranean Sea.
As shown in Sect. 2.3, deviating observation intervals and/or calculation methods (in the case of $T_{\text {avg }}$ ) occasionally cause considerable differences of the daily station data used for the two data sets (mainly ECA\&D for E-OBS, mainly SYNOP for DecReg), especially in winter. The corresponding comparison (SYNOP minus ECA\&D at stations with identical coordinates) for the investigated day is shown in Fig. 20d. The differences of the grid data found in eastern Europe are well explained by the deviating source data. In contrast, the station data in the Mediterranean region are found consistent for this day. Here, differences in the assumed lapse rates lead to varying results for grid cells of high altitudes. Since in E-OBS these dependencies are calculated locally (see Haylock et al., 2008), there is a potentially better reflection of small-scale changes; however, this strategy lacks robustness in cases of missing representation by local station data. The maps of mean daily deviation (Fig. 20e) and mean absolute daily deviation (Fig. 20f) between DecReg and E-OBS indicate persistent differences in mountain regions around the Mediterranean.

To evaluate the temperature grid data in mountain regions, a comparison with ERA-Interim reanalysis temperatures at $850 \mathrm{hPa}$ (http://apps.ecmwf.int/datasets/data/ interim-full-daily/; Dee et al. (2011)) was made. Daily mean model fields were calculated from the 6-hourly raw data by 

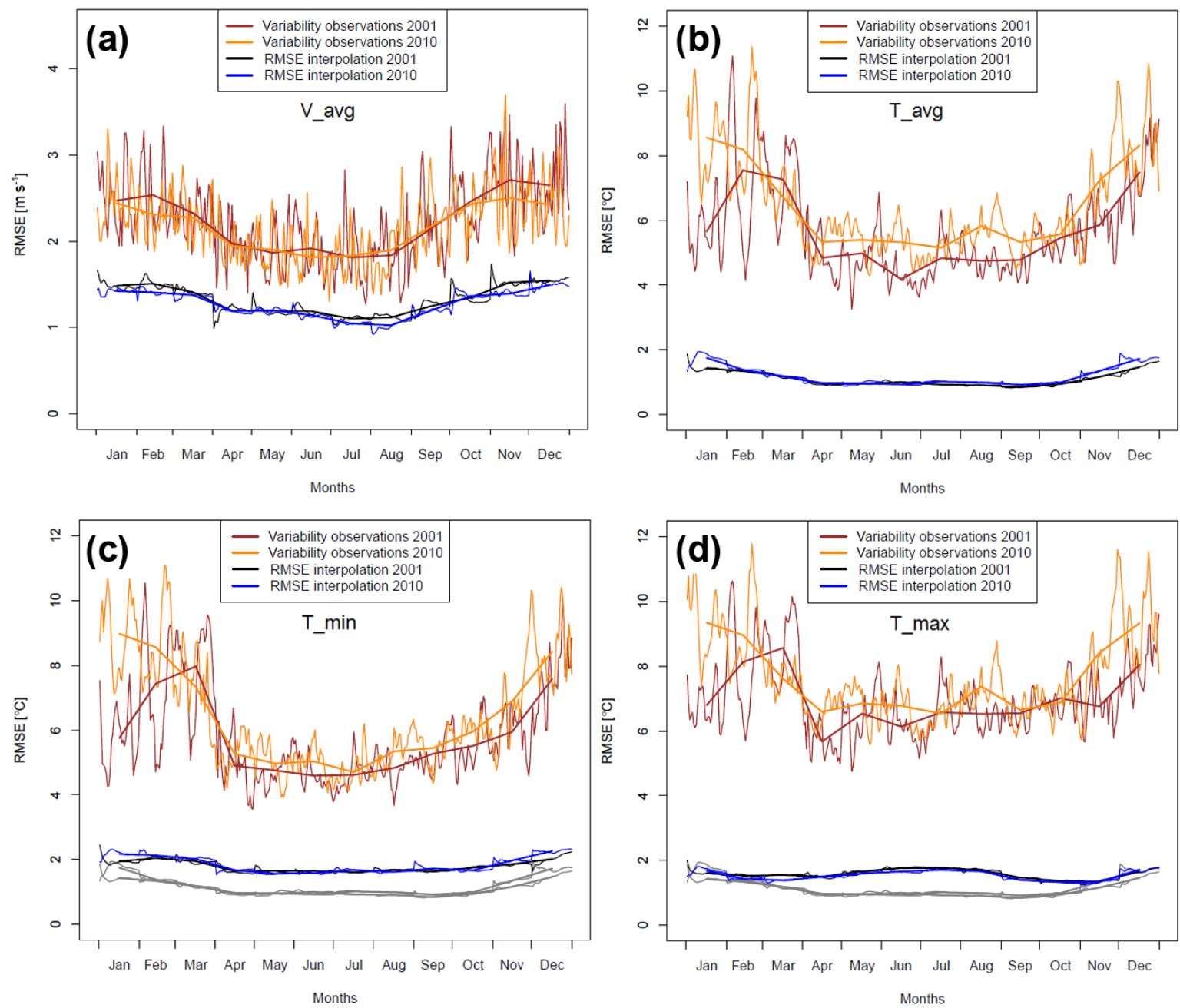

Figure 18. Annual cycle of daily RMSE according to cross validation for 2001 (black) and 2010 (blue) for (a) mean wind speed $V_{\text {avg }}$, (b) mean temperature $T_{\mathrm{avg}}$, (c) minimum temperature $T_{\min }$ and (d) maximum temperature $T_{\max }$. For comparison, the daily standard deviation over all station observations is displayed in brown (2001) and orange color (2010). Corresponding curves of monthly means are added to all data. In panels (c) and (d) the RMSE data for $T_{\mathrm{avg}}$ are displayed in grey color for comparison.

averaging in the same way, as described in Sect. 2.2. The data fields were interpolated to the E-OBS and the DecReg grid using bilinear remapping. All grid points at altitudes near the level of $850 \mathrm{hPa}$ - a range between 1200 and $1800 \mathrm{~m}$ was defined - were compared with ERA-Interim. To correct for temperature differences caused by height deviations, observational grid data were normalized (for each day and grid point) to the actual geopotential height at $850 \mathrm{hPa}$ (from daily ERA-Interim field) by assuming linear lapse rates determined on the basis of the eight surrounding grid cells.

The outcomes of this comparison for January 2010 are shown in Fig. 21. For the difference E-OBS minus ERAInterim at $850 \mathrm{hPa}$ (Fig. 21a), mean daily deviations vary between -3 and $+8 \mathrm{~K}$. A clear overestimation of mountain temperatures is indicated for the Atlas Mountains, the Sierra Nevada (southern Spain) and for the French Alps. Consis- tency with ERA-Interim is high in Turkey, central and northern Spain and parts of the Alps. Mountain data in Norway and Romania tend to be underestimated for most of the days. The DecReg data (Fig. 21b) are very consistent with ERAInterim in the Alps, the mountains in southeastern Europe and in Iceland. However, significant negative biases of up to $-5 \mathrm{~K}$ are indicated for, e.g., the Atlas and Scandinavian mountains and elevated areas of the Middle East. For eight grid points (encircled in Fig. 21a and b) a daily comparison was made for the entire year 2010. Corresponding time series of the monthly mean of daily deviations from ERA-Interim are displayed for E-OBS (Fig. 21c) and DecReg (Fig. 21d). Considerable discrepancies partly exceeding $5 \mathrm{~K}$ are diagnosed for the E-OBS grid points in Morocco, the Sierra Nevada and the French Alps (compare legend and color code of circles in Fig. 21a and b) throughout the year. At the other 


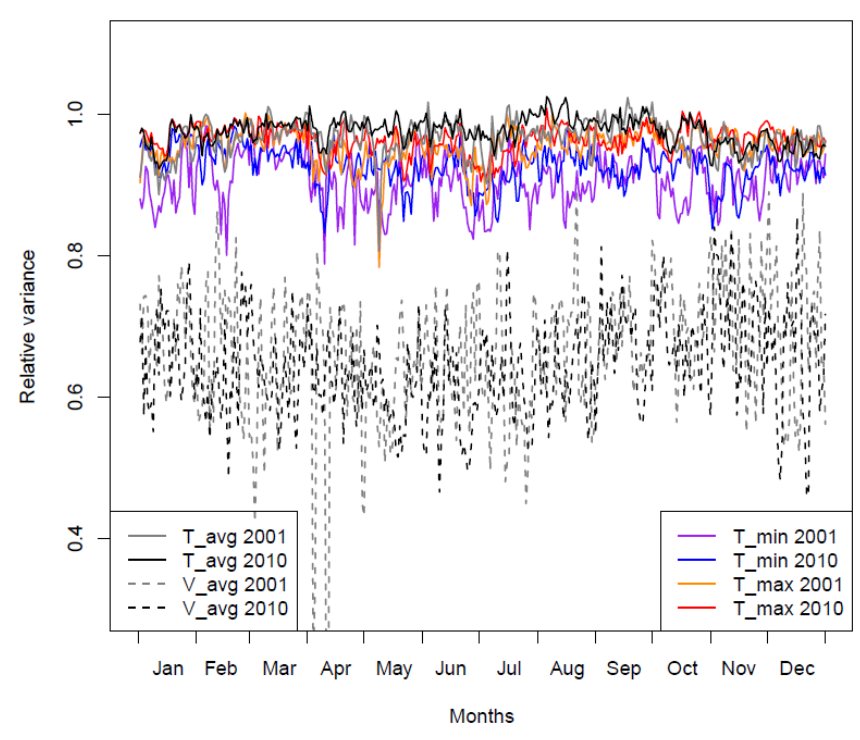

Figure 19. Time series of the ratio of spatial variance of interpolated vs. observed station data for the years 2001 and 2010 based on cross validation. The color code indicates the parameters and years.

single grid points the consistency between E-OBS and ERAInterim is relatively high, with only occasional negative biases above $3 \mathrm{~K}$ occurring in winter months. The DecReg data show constant negative biases compared with ERA-Interim of around $2-3 \mathrm{~K}$ at the grid points in Morocco, Spain and Syria. For the other mountain regions the data agree relatively well, except for single winter months with clear negative biases.

In a further analysis the ERA-Interim temperatures at $850 \mathrm{hPa}$ were compared with SYNOP station data to evaluate the representativeness of the model data (initial resolution of $\approx 80 \mathrm{~km})$ for the relatively fine grids of E-OBS $(\approx 25 \mathrm{~km})$ and DecReg $(\approx 5 \mathrm{~km})$. The data of up to three suitable mountain stations (heights between 1200 and $1800 \mathrm{~m}$ ) within a radius of $2.5^{\circ}(\approx 280 \mathrm{~km})$ around the single grid points analyzed above were compared with the nearest ERA-Interim grid point (interpolated; $0.044^{\circ}$ ). Similarly to the procedure above, station temperatures were normalized to the $850 \mathrm{hPa}$ geopotential height for each day (based on lapse rates determined from DecReg). The results of this comparison in the environment of the single grid points are shown in Fig. 21e. The consistency between station data and ERA-Interim (no station data available for grid points in Spain) is very high for most time of the year 2010. Daily deviations are typically within the range of $\pm 2 \mathrm{~K}$. For the grid points in Norway and Turkey, similar negative biases are observed as for E-OBS and DecReg for the winter months (Fig. 21c and d). Thus, the reanalysis data tend to be incorrect or not representative in some of the mountain regions during winter. Reversely, the observational grid data of E-OBS and DecReg are indicated to be consistent with the observations for these areas, even in winter.
Overall, E-OBS and DecReg mountain temperatures at around $1500 \mathrm{~m}$ are in an acceptable agreement with ERAInterim reanalysis data and station observations. However, the interpolation procedure used for E-OBS fails to reproduce temperature changes with altitude sufficiently in areas without suitable observations. The regression approach presented in this study is indicated to be slightly more reliable in these problematic areas but is incapable of representing local deviations from the lapse rate determined for each region. Additionally, both approaches are incapable of dealing with nonlinear lapse rates.

Regarding the observation intervals of the daily E-OBS data, no consistency throughout the domain is ensured, which is a result of deviating procedures applied by the national weather agencies providing data to ECA\&D. The SYNOP input data used in DecReg are based on the same daily intervals. Thus, potential discontinuities of temperature fields near national borders are avoided and comparability with model data for defined intervals ensured.

Apart from the causes discussed above, differences in the distribution of the input station data used in E-OBS and DecReg can also lead to deviating grid data. This aspect is important for regions where the density of stations is generally low, as observed around the Mediterranean Sea, especially in the early years of the decade (compare Fig. 1).

\subsection{Evaluation of uncertainty estimates}

As mentioned in Sect. 4.3, uncertainty fields based on regression error and kriging variance were determined for each monthly and daily data field. In the following, a comparison of these estimates with the findings from cross validation is presented. We use daily cross-validation results (as shown in, for example, Fig. 16) and the monthly mean of daily IQR at the nearest adjacent grid points. For each station the number of interpolated data within the IQR error interval is counted for the 2 example months January and July 2010. The results of this experiment are displayed in Fig. 22 for daily mean temperature. Blue colors indicate point data for which more daily data lie outside the range of error than expected. Grey dots mark data for which the monthly statistic fairly agrees with the definition of IQR $(50 \pm 10 \%)$. Locations with more accurate data than indicated by the IQR are colored red. Additionally, corresponding frequency distributions are displayed for the 2 months (Fig. 22a2, b2).

The outcomes for $T_{\text {avg }}$ show that IQR uncertainty levels are, on average, relatively consistent with the crossvalidation results. Nevertheless, small-scale changes of the uncertainty, as for mountainous areas, are not well reflected in the data fields of IQR. As a consequence, a tendency towards overestimation of the error in topographically homogeneous regions is observed, while in regions with complex terrain errors tend to be underestimated. For July 2010 the distribution is less symmetric than for January 2010. As noted in Sect. 4.3, local changes of uncertainty as a result 

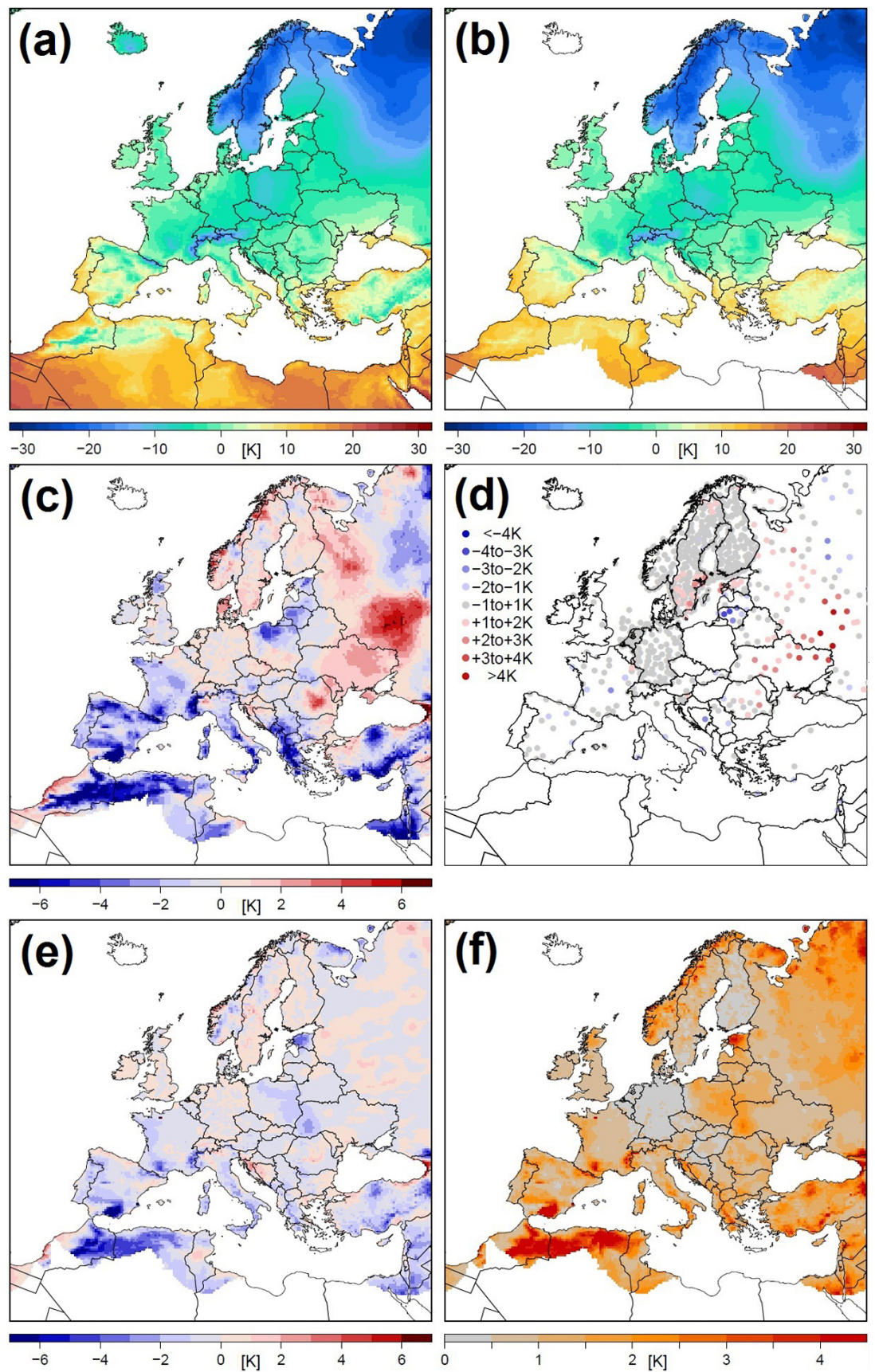

Figure 20. Comparison of daily temperature grid data from DecReg and E-OBS for January 2010: (a) DecReg daily mean temperature ( $\left.T_{\text {avg }}\right) 31$ January 2010; (b) E-OBS $T_{\text {avg }} 31$ January 2010; (c) DecReg minus E-OBS $T_{\text {avg }} 31$ January 2010; (d) SYNOP minus ECA\&D

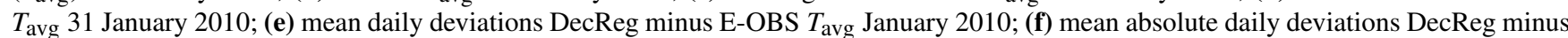
E-OBS $T_{\text {avg January }} 2010$.

of a relatively low or relatively high local variability of the input data are not considered in the kriging variance. This measure mainly depends on the variogram parameters - reflecting average changes of the correlation between adjacent data values with distance - and the station density. For certain months the basic variogram parameters determined for each region ("null" variogram, see Sect. 3.2) can deviate sig- nificantly from the real data characteristics in that specific month. The restriction of the automated variogram fitting (to avoid unstable variogram models, Sect. 3.2) can lead to mean IQR estimates deviating from the mean errors based on cross validation.

For wind speed (Fig. 23) qualitatively similar results are indicated. On average, the IQR defines a reasonable uncer- 

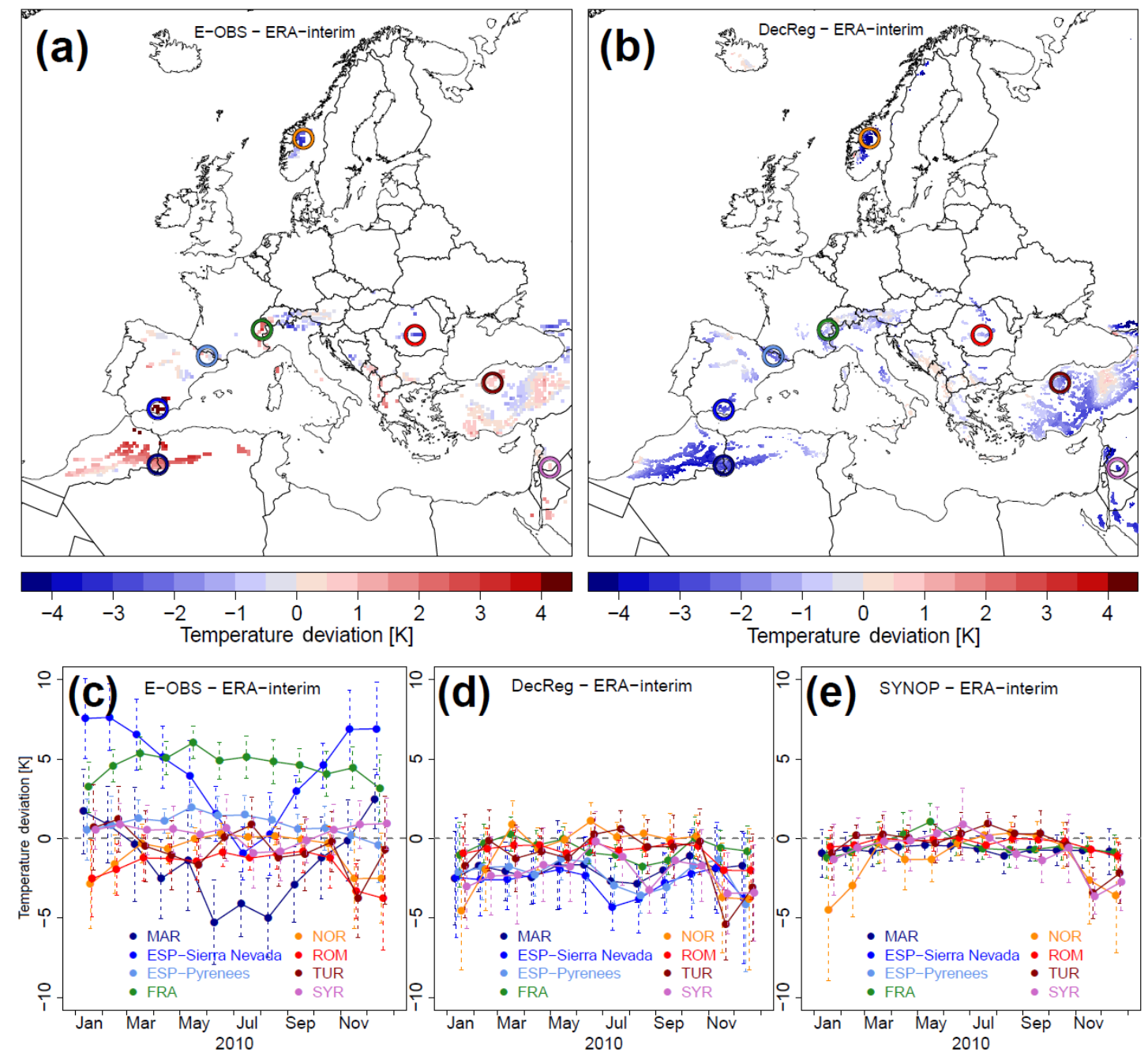

Figure 21. Comparison of daily temperature grid data at altitudes of around $1500 \mathrm{~m}$ from DecReg and E-OBS with ERA-Interim $850 \mathrm{hPa}$ temperatures for January 2010: (a) E-OBS minus ERA-Interim mean deviations of daily mean temperatures $\left(T_{\text {avg }}\right)$; (b) DecReg minus ERAInterim mean deviations of daily $T_{\mathrm{avg}}$; (c) annual cycle of E-OBS minus ERA-Interim deviations of daily $T_{\text {avg }}$ (monthly mean and standard deviation) at single grid points encircled in panel (a); (d, e) same as in panel (c) but for the comparison DecReg minus ERA-Interim and SYNOP minus ERA-Interim, respectively.

tainty range. However, also here the spatial variation is very high. In contrast to temperature, the distribution of significantly outlying data values is less systematic for wind speed (Fig. 23a2, b2). This can be explained with its relatively high spatial variability on small scales.

\section{Summary}

In this work interpolation schemes for daily station data of minimum, maximum and mean temperature as well as daily mean wind speed in $0.044^{\circ}(\approx 5 \mathrm{~km})$ resolution for Europe (rotated grid, virtual North Pole at $39.25^{\circ} \mathrm{N}, 162^{\circ} \mathrm{W}$ ) are presented. To achieve a high data consistency, temperature extremes are based on the same $12 \mathrm{~h}$ intervals of night (18:00 to $06: 00$ UTC) and day (06:00 to 18:00 UTC), and a consis- tent $24 \mathrm{~h}$ interval starting at 00:00 UTC is used for the calculation of daily means. A regression kriging approach using predictors altitude, continentality and zonal monthly mean temperature, based on the work by Krähenmann et al. (2011), is applied for the temperature parameters. Modifications and further developments were implemented to adapt the existing routine to the special demands of our project. For wind speed a new regression kriging procedure involving the predictor variables exposure, coastal distance, roughness length and $850 \mathrm{hPa}$ ERA-Interim reanalysis wind speeds was developed.

As an important prerequisite for the interpolation, a preprocessing to derive daily means from hourly SYNOP data in combination with a profound quality control was established. Also for the other input data, daily extreme temper- 

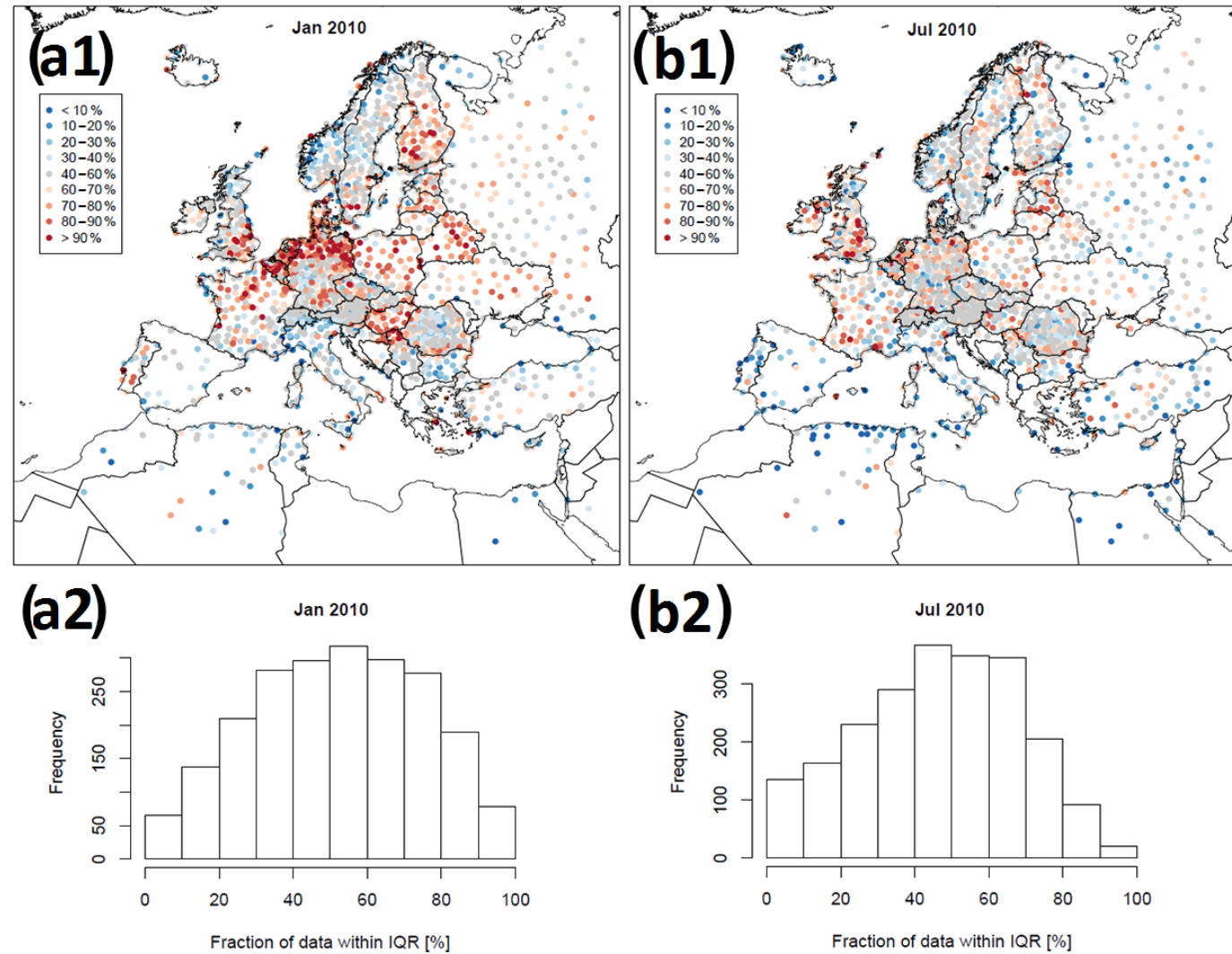

(b2)

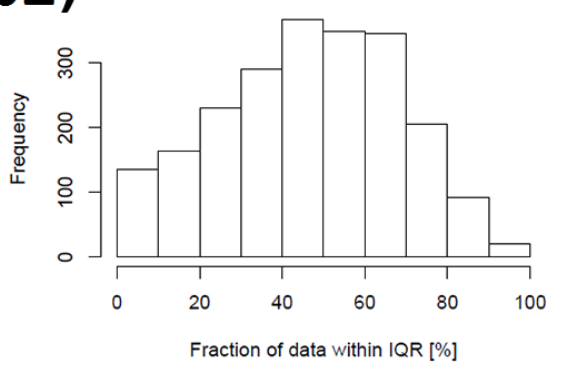

Figure 22. Comparison of cross-validation results with gridded uncertainty estimates for daily mean temperature in January 2010 (a1) and July 2010 (b1): fraction of interpolated daily data at stations within nearest-neighbor gridded IQR (interquartile range). Blue indicates an underestimation; red indicates an overestimation of the uncertainty. In panels (a2) and (b2) corresponding frequency distributions are displayed.

atures and the data of the ECA\&D archive, detailed quality control procedures were developed. In order to maintain consistency with SYNOP, a selection algorithm, controlling the integration of ECA\&D data in regions where SYNOP data are sparse and consistency between the two sources is high, was implemented.

For the time period 2001-2010 the spatial variation of the monthly means can be well explained by the predictors. We obtain relative explained variances in the range of 80-90\% for the temperature parameters and about $50-60 \%$ for wind speed.

Cross validation is performed for the years 2001 and 2010 to assess the quality of the daily interpolation products. For daily mean temperature, RMSEs of about $1-2 \mathrm{~K}$ are diagnosed. The accuracy for the daily extremes is typically lower, with values around or slightly below $2 \mathrm{~K}$. In winter interpolation accuracies tend to be reduced compared to summer. For daily maximum temperatures an additional summer reduction in gridding accuracy is detected. The RMSE for daily mean wind speed lies in the range of $1-1.5 \mathrm{~m} \mathrm{~s}^{-1}$. Here, an annual cycle, with the higher values occurring in winter, is also indicated.
Concerning the conservation of spatial variance, very good performance is found for the temperature parameters. In the interpolation products $90-100 \%$ of the observed variance is typically preserved. Only for minimum temperature are at times lower values recorded. For daily wind speed, a fraction of $60-80 \%$ of the original variance is preserved after interpolation. The relatively high degree of unexplained small-scale variance leads to a smoothing of the wind data.

The cross-validation results are also used to evaluate the quality of the gridding uncertainty based on kriging variance and regression errors. On average, a reasonable consistency between these data is found. Nevertheless, temporal and spatial variations of uncertainty occurring on small scales are not adequately reflected in the gridded uncertainties.

In comparison with the E-OBS temperature data occasional discrepancies of more than $5 \mathrm{~K}$ between the two data sets occur. These are caused, on one hand, by countryspecific deviations in the daily observation intervals and, in certain regions, by a different availability of the input data. On the other hand, different approaches to derive lapse rates lead to larger deviations in mountain regions. Both data sets fail to match ERA-Interim reanalysis temperatures at 


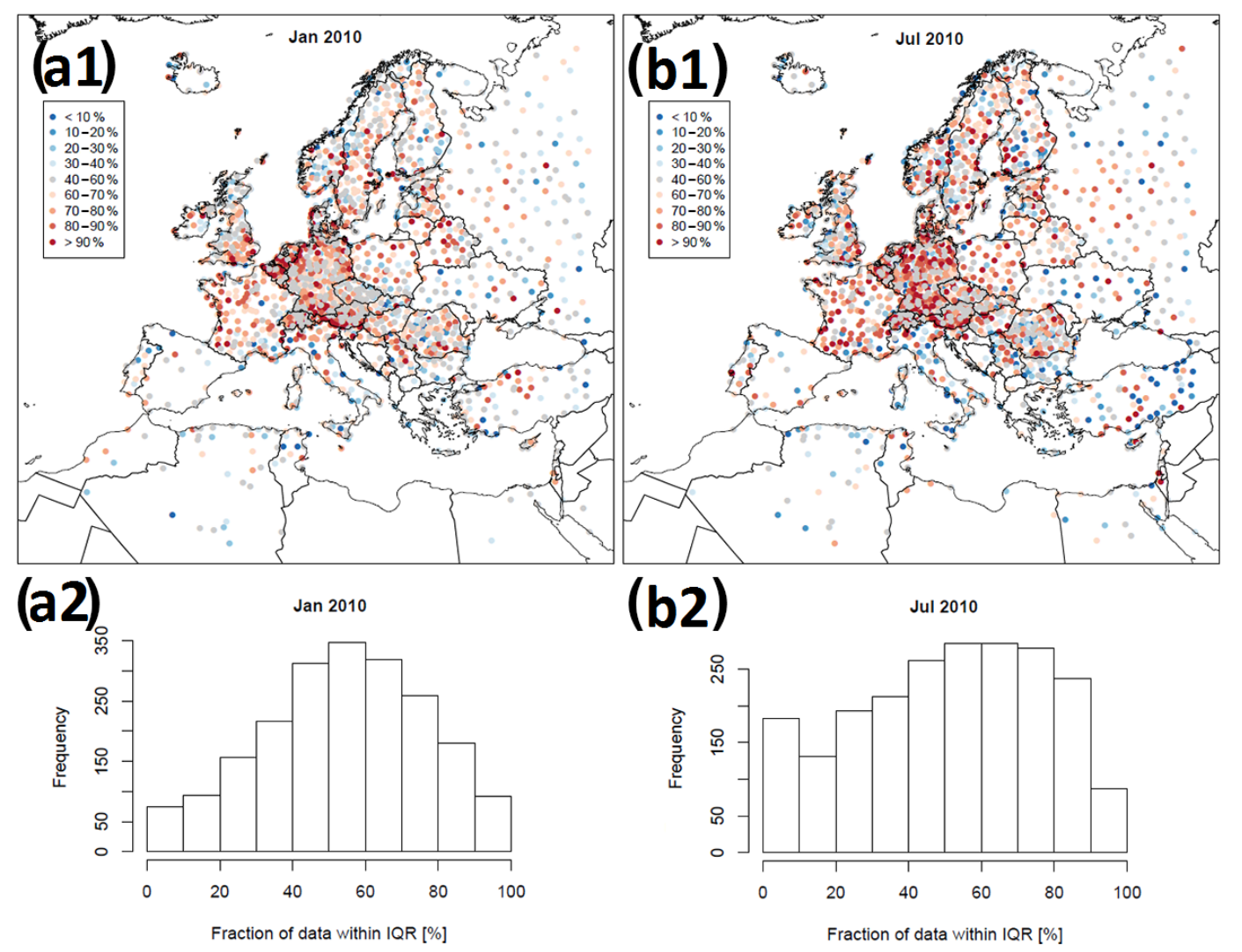

Figure 23. Comparison of cross-validation results with gridded uncertainty estimates for daily mean wind speed in January 2010 (a1) and July 2010 (b1): fraction of interpolated daily data at stations within nearest-neighbor gridded IQR (interquartile range). Blue indicates an underestimation; red indicates an overestimation of the uncertainty. In panels (a2) and (b2) corresponding frequency distributions are displayed.

$850 \mathrm{hPa}$ satisfactorily in areas without sufficient data from mountain stations.

\section{Conclusions}

The regression kriging approaches used in this work for the interpolation of daily temperature and wind speed observations on a grid size of $0.044^{\circ}(\approx 5 \mathrm{~km})$ show good performance in terms of accuracy and variance preservation. With the inclusion of suitable predictor variables small-scale characteristics of the meteorological parameters can be well captured.

For the dependency of temperature on altitude more reliable regression results are obtained by performing this regression separately and on the basis of representative stations. Also, day-to-day variations of this dependency are considered in the new setup used in this study. Nevertheless, the linear regression approach applied to the relatively large areas of each region is not capable to reflect nonlinear vertical temperature changes and spatial differences of this parameter within a region. More complex approaches considering this issue in the calculation of high-resolution grid data in mountainous regions have been published (e.g., Frei, 2013). However, these specialized strategies require the presence of stations representative for a certain area and altitude level. For the relatively large domain dealt with in our work, where many mountain regions are insufficiently represented by station data, the inclusion of radiosonde data might offer a promising strategy in this respect.

Concerning the regression of wind speed, a considerable part of spatial variance on a monthly basis (40-50\%) remains unexplained by the predictors used in this work. For predictor fields of exposure, coastal distance and roughness length it would be more realistic to take into account the current wind direction and local predictor conditions determined for this wind direction. This strategy would introduce further complexity in the calculations. However, the percentage of variance explained by predictors as well as the final interpolation accuracy could likely be increased.

The gridded error estimates calculated for the daily and monthly products are, on regional average, reasonable, but for certain days and areas these estimates are found to be unrealistic. An alternative approach yielding more reliable errors (Yamamoto, 2000) was not implemented due to the 
enormous increase in computing time. Thus, the determination of accurate uncertainty estimates remains an issue for data sets of high resolution in space and time.

However, users of these grid data are recommended to consider the IQR uncertainty fields provided in separate files in their analyses. Especially in parts of North Africa the uncertainties are usually very high due to very sparse observations. To deal with this issue, IQR thresholds tolerable for a specific analysis could be defined to exclude regions with less reliable data.

\section{Data availability}

The data sets presented in this article are published at doi:10.5676/DWD_CDC/DECREG0110v2 (Brinckmann and Bissolli, 2016). Elevation data of the DecReg grid are available at ftp://ftp-cdc.dwd.de/pub/CDC/help/.

Acknowledgements. The authors would like to acknowledge the Bundesministerium für Bildung und Forschung (BMBF) for funding this project. The colleagues in the department Regional Climate Monitoring at Deutscher Wetterdienst (DWD) are acknowledged, especially Maya Körber and Andrea Kreis for their support in technical issues related to UNIX and database queries. We also thank our partners participating in the DecReg project for the fruitful cooperation during the 26 months. Furthermore, the E-OBS data set from the EU-FP6 project ENSEMBLES (http://ensembles-eu.metoffice.com) and the data providers in the ECA\&D project are acknowledged (http://www.ecad.eu).

Edited by: G. König-Langlo

Reviewed by: two anonymous referees

\section{References}

Ahmed, S. and de Marsily, G.: Comparison of geostatistical methods for estimating transmissivity using data on transmissivity and specific capacity, Water Resour. Res., 23, 1717-1737, doi:10.1029/WR023i009p01717, 1987.

Bartholome, E. and Belward, A. S.: GLC2000: a new approach to global land cover mapping from Earth observation data, Int. J. Remote Sens., 26, 1959-1977, doi:10.1080/01431160412331291297, 2005.

Brinckmann, S. and Bissolli, P.: DecReg/MiKlip gridded data of near-surface temperature $(2 \mathrm{~m})$ and wind speed $(10 \mathrm{~m})$ in Europe for the period 2001-2010, Version v002, DWD Climate Data Center, doi:10.5676/DWD_CDC/DECREG0110v2, 2016.

Dee, D. P., Uppala, S. M., and Simmons, A. J. E. A.: The ERAInterim reanalysis: configuration and performance of the data assimilation system, Q. J. Roy. Meteor. Soc., 137, 553-597, doi:10.1002/qj.828, 2011.

Deutsch, C. V. and Journel, A.: GSLIB: Geostatistical Software Library and User's Guide, 2nd edn., Oxford University Press, New York, USA, 1998.
Frei, C.: Interpolation of temperature in a mountainous region using nonlinear profiles and non-Euclidean distances, Int. J. Climatol., 34, 1585-1605, doi:10.1002/joc.3786, 2013.

Gerth, W.-P. and Christoffer, J.: Windkarten von Deutschland, Meteorol. Z., 3, 67-77, 1994.

Gorczynski, L.: Sur le calcul du degré de continentalisme et son application dans la climatologie, Geografiska Annaler, 2, 324331, 1920.

Hansen, J., Ruedy, R., Sato, M., and Lo, K.: Global surface temperature change, Rev. Geophys., 48, RG4004, doi:10.1029/2010RG000345, 2010.

Haylock, M. R., Hofstra, N., Klein Tank, A. M. G., Klok, E. J., Jones, P. D., and New, M.: A European daily highresolution gridded data set of surface temperature and precipitation for 1950-2006, J. Geophys. Res., 113, D20119, doi:10.1029/2008JD010201, 2008.

Hogewind, F. and Bissolli, P.: Operational maps of monthly mean temperature for WMO Region VI (Europe and Middle East), Idojaras, 115, 31-49, 2011.

Hofstra, N., Haylock, M. R., New, M., Jones, P. D., and Frei, C.: Comparison of six methods for the interpolation of daily European climate data, J. Geophys. Res., 113, D21110, doi:10.1029/2008JD010100, 2008.

Holton, J. R. and Hakim, G. J.: An Introduction to Dynamic Meteorology, 5th edn., Academic Press, Waltham, MA, USA, 2012.

Klein Tank, A. M. G., Wijngaard, J. B., Können, G. P., Böhm, R., Demarée, G., Gocheva, A., Mileta, M., Pashiardis, S., Hejkrlik, L., Kern-Hansen, C., Heino, R., Bessemoulin, P., MüllerWestermeier, G., Tzanakou, M., Szalai, S., Pálsdóttir, T., Fitzgerald, D., Rubin, S., Capaldo, M., Maugeri, M., Leitass, A., Bukantis, A., Aberfeld, R., van Engelen, A. F. V., Forland, E., Mietus, M., Coelho, F., Mares, C., Razuvaev, V., Nieplova, E., Cegnar, T., Antonio López, J., Dahlström, B., Moberg, A., Kirchhofer, W., Ceylan, A., Pachaliuk, O., Alexander, L. V., and Petrovic, P.: Daily dataset of 20th-century surface air temperature and precipitation series for the European Climate Assessment, Int. J. Climatol., 22, 1441-1453, doi:10.1002/joc.773, 2002.

Krähenmann, S. and Ahrens, B.: Spatial gridding of daily maximum and minimum 2-m temperatures supported by satellite observations, Meteorol. Atmos. Phys., 120, 87-105, doi:10.1007/s00703-013-0237-9, 2013.

Krähenmann, S., Bissolli, P., Rapp, J., and Ahrens, B.: Spatial gridding of daily maximum and minimum temperatures in Europe, Meteorol. Atmos. Phys., 114, 151-161, doi:10.1007/s00703011-0160-x, 2011.

Li, J. and Heap, A. D.: A review on spatial interpolation methods for environmental scientists, Geoscience Australia, 15, 585-596, doi:10.1127/0941-2948/2006/0162, 2008.

Luo, W., Taylor, C., and Parker, S. R.: A comparison of spatial interpolation methods to estimate continuous wind speed surfaces using irregularly distributed data from England and Wales, Int. J. Climatol., 28, 947-959, doi:10.1002/joc.1583, 2008.

Matheron, G.: Principles of geostatistics, Econ. Geol., 58, 12461266, 1963.

Mitchell, T. D. and Jones, P. D.: An improved method of constructing a database of monthly climate observations and associated highresolution grids, Int. J. Climatol., 25, 693-712, doi:10.1002/joc.1181, 2005. 
Morice, C. P., Kennedy, J. J., Rayner, N. A., and Jones, P. D.: Quantifying uncertainties in global and regional temperature change using an ensemble of observational estimates: the HadCRUT4 data set, J. Geophys. Res., 117, D08101, doi:10.1029/2011JD017187, 2012.

Odeh, I. O. A., McBratney, A. B., and Chittleborough, D. J.: Further results on prediction of soil properties from terrain attributes: heterotropic cokriging and regression-kriging, Geoderma, 67, 215226, doi:10.1016/0016-7061(95)00007-B, 1995.

Pebesma, E. J.: Multivariable geostatistics in $S$ : the gstat package, Comput. Geosci., 30, 683-691, doi:10.1016/j.cageo.2004.03.012, 2004.

Rohde, R., Muller, R., Jacobsen, R., Perlmutter, S., Rosenfeld, A., Wurtele, J., Curry, J., Wickham, C., and Mosher, S.: Berkeley Earth Temperature Averaging Process, Geoinfor. Geostat: An Overview 1, 1-13, doi:10.4172/2327-4581.1000103, 2013.

Sanderson, M.: The classification of climates from Pythagoras to Koeppen, B. Am. Meteorol. Soc., 80, 669-673, doi:10.1175/1520-0477(1999)080<0669:TCOCFP>2.0.CO;2, 1999.

Smith, T. M., Reynolds, R. W., Peterson, T. C., and Lawrimore, J.: Improvements to NOAA's historical merged land-ocean surface temperature analysis (1880-2006), J. Climate, 21, 2283-2296, doi:10.1175/2007JCLI2100.1, 2008.
Stahl, K., Moore, R. D., Floyer, J. A., Asplin, M. G., and McKendry, I. G.: Comparison of approaches for spatial interpolation of daily temperature in a large region with complex topography and highly variable station density, Agr. Forest Meteorol., 139, 224-236, doi:10.1016/j.agrformet.2006.07.004, 2006.

van den Besselaar, E. J. M., Klein Tank, A. M. G., van der Schrier, G., and Jones, P. D.: Synoptic messages to extend climate data records, J. Geophys. Res., 117, D07101, doi:10.1029/2011JD016687, 2012.

Walter, A., Keuler, K., Jacob, D., Knoche, R., Block, A., Kotlarski, S., Müller-Westermeier, G., Rechid, D., and Ahrens, W.: A high resolution reference data set of German wind velocity 1951-2001 and comparison with regional climate model results, Meteorol. Z., 23, 585-596, 2006.

Yamamoto, J. K.: An alternative measure of the reliability of ordinary kriging estimates, Math. Geol., 32, 489-509, doi:10.1023/A:1007577916868, 2000. 